\title{
Evaluation of PSE as a model for supersonic jet using transfer functions
}

\author{
Vitor G. Kleine, Kenzo Sasaki ${ }^{\dagger}$ and André V. G. Cavalieri ${ }^{\ddagger}$ \\ Instituto Tecnológico de Aeronáutica, São José dos Campos, SP, 12228-900, Brazil \\ Guillaume A. Brès ${ }^{\S}$ \\ Cascade Technologies Inc., Palo Alto, CA 94303 \\ Tim Colonius $₫$ \\ California Institute of Technology, Pasadena, CA 91125
}

\begin{abstract}
Parabolized Stability Equations (PSE) have been shown to model wavepackets and, consequently, the near field of turbulent jets with reasonable accuracy. Because of these capabilities, PSE is a promising reduced-order model to derive control laws that could be employed to reduce the sound generation of a jet. The purpose of this work is to apply PSE to obtain time-domain transfer functions that could estimate both the fluid-dynamic and the acoustic fields of a supersonic jet. The results of this model were compared to results obtained from a database of a well-validated large-eddy simulation of a supersonic jet.

Based on the unsteady pressure data at a input position, the time-domain pressure field was estimated using transfer functions obtained using PSE and an empirical method based on the LES data. The prediction scheme employed is a single-input-single-output (SISO), linear model. The unsteady pressure predicted by PSE showed good agreement with the LES results, especially if the input position is outside the mixing layer. For this region, the prediction capabilities of PSE are comparable to those of empirical transfer functions. The agreement is good even for output points taken in the acoustic field, showing that it is possible to estimate the time-domain behaviour of Mach-wave radiation using transfer functions.

This indicates that PSE could not only be used to predict the sound generation, but also to open up new potentialities to attenuate noise by means of closed-loop control of the flow. The exploration of the regions where the method displayed good agreement, presented in this work, can guide the positioning of sensors and actuators for experimental implementation of closed-loop control in a jet.
\end{abstract}

\section{Introduction}

Parabolized Stability Equations (PSE) have been shown to model frequency-domain wavepackets of turbulent subsonic jets with reasonable accuracy. ${ }^{1,2}$ Since wavepackets correlate well with sound radiation, ${ }^{2}$ PSE can be applied to indirectly study the sound radiation of subsonic jets. Due to its accuracy, PSE was applied to obtain time-domain transfer functions that relate the unsteady data of two separated positions of a subsonic jet. ${ }^{3,4}$ It was shown that the transfer functions derived in previous works using PSE are comparable to other more computationally expensive methods based on empirical data. Hence, these transfer functions are promising tools to derive control laws that could be employed to reduce the sound generation of a jet. An initial attempt, targeting the Kelvin-Helmholtz instability of a two-dimensional mixing layer, has shown promising results. ${ }^{4}$ The low-computational cost of the PSE transfer functions also allows its use for the

*Assistant Professor, Division of Aeronautical Engineering

${ }^{\dagger} \mathrm{PhD}$ Student, Division of Aeronautical Engineering

¥Professor, Division of Aeronautical Engineering

$\S$ Senior Research Scientist

TProfessor, Dept. of Mechanical Engineering. 
study of the most appropriate areas for sensor/actuator placement for the experimental implementation of closed-loop control.

In the same sense, in the case of an ideally expanded supersonic jet, besides near-field wavepackets, PSE has also been used to directly predict the acoustic field, which is dominated by Mach-wave radiation. ${ }^{5}$ This study shows encouraging frequency-domain predictions, which are compared to data from a large-eddy simulation (LES).

The purpose of this work is to investigate the use of PSE to obtain time-domain transfer functions modeling both the dynamic and the acoustic fields of a supersonic jet. These capabilities would indicate that PSE could not only be used to predict the sound generation but also to open up new potentialities to attenuate noise by means of closed-loop control of the flow. The transfer functions and the prediction data were compared to empirical transfer functions and results obtained from a database of a large-eddy simulation (LES) of an isothermal ideally-expanded supersonic jet. ${ }^{6,7}$

\section{Model}

\section{A. Parabolised Stability Equations}

The PSE represents an extension of the parallel-flow linear stability problem to base-flows with mild streamwise variation. The total flow field $\mathbf{q}$ is decomposed into a mean, time-averaged axisymmetric component $\overline{\mathbf{q}}(\mathbf{x})$ and a time dependent fluctuation $\mathbf{q}^{\prime}(\mathbf{x}, t)$, where $\mathbf{q}=\left[u_{x}, u_{r}, u_{\theta}, \rho, T\right]^{T}$ represents the flow variables (axial, radial \& azimuthal components of velocity, density and temperature, respectively) and cylindrical coordinates $\mathbf{x}=[x, r, \theta]$ are considered.

Since the mean flow is homogeneous in the azimuthal direction and in time, the fluctuation may be expressed in terms of normal modes in these directions.

$$
\mathbf{q}^{\prime}(\mathbf{x}, t)=\sum_{\omega} \sum_{m} \tilde{\mathbf{q}}(x, r) \mathrm{e}^{\mathrm{i} m \theta} \mathrm{e}^{-\mathrm{i} \omega t},
$$

where $\tilde{\mathbf{q}}$ is the complex amplitude for frequency $\omega$ and azimuthal wavenumber $m$.

One may further simplify the solution by decomposing $\tilde{\mathbf{q}}(x, r)$ into two separate contributions, a slowly varying function representing the amplitude envelope of the solution and a fast, oscillatory function that captures the wave-like axial structure. This solution corresponds to the wavepacket Ansatz for slowly diverging jets $^{8}$ and allows parabolisation of the linearised equations,

$$
\mathbf{q}^{\prime}(\mathbf{x}, t)=\hat{\mathbf{q}}(x, r) \mathrm{e}^{\mathrm{i} \int^{x} \alpha\left(x^{\prime}\right) \mathrm{d} x^{\prime}} \mathrm{e}^{\mathrm{i} m \theta} e^{-\mathrm{i} \omega t},
$$

where $\alpha=\alpha_{r}+\mathrm{i} \alpha_{i}$ is the complex axial wavenumber, whose imaginary part is related to exponential growth or decay of disturbances in $x$. Slow variations in $x$ are assumed for both $\hat{\mathbf{q}}$ and $\alpha$. The decomposition in eq. (2) is based on a distinction between the slowly-varying amplitude functions $\hat{\mathbf{q}}(x, r)$ and the fast changes associated with the $\alpha$ integral.

On substitution of equation 2 into the linearised, compressible Euler, energy and continuity equations, a matrix system is obtained,

$$
(A(\overline{\mathbf{q}})+B(\overline{\mathbf{q}}, \alpha, \omega)) \hat{\mathbf{q}}+C(\overline{\mathbf{q}}) \frac{\partial \hat{\mathbf{q}}}{\partial x}+D(\overline{\mathbf{q}}) \frac{\partial \hat{\mathbf{q}}}{\partial r}=0,
$$

where the high Reynolds number of the flows considered justifies neglect of the viscous terms. Details of the derivation may be found elsewhere. ${ }^{9,10}$ The linearity of the above system allows independent calculation of each $\omega-m$ combination, an ensemble of which is then used to construct a frequency-domain model, from which a Transfer Function between any two given positions in the jet can be derived.

The PSE system (3) requires a further condition, necessary to eliminate the ambiguity between $\hat{\mathbf{q}}$ and $\alpha$, since the spatial growth in the axial direction may be absorbed into either the shape function $\hat{\mathbf{q}}$ or the complex amplitude $\mathrm{e}^{\mathrm{i} \int \alpha\left(x^{\prime}\right) d x^{\prime}}$. We follow the normalisation proposed in Herbert et al. ${ }^{9}$ which removes the exponential axial dependence of the shape functions by imposing,

$$
\int_{0}^{\infty} \hat{\mathbf{q}} \frac{\partial \hat{\mathbf{q}} *}{\partial x} r \mathrm{~d} r=0
$$

Discretising equations 3 and 4 one obtains, via an iterative algorithm, ${ }^{1}$ a numerical solution for $\hat{\mathbf{q}}$ and $\alpha$. Further discussion on this procedure and on the PSE method in general can be found in the literature. ${ }^{1,2,9-11}$ 


\section{Numerical Methods}

A pseudo-spectral method is used to discretise the functions in the radial direction by means of Chebyshev polynomials, whose spectral accuracy is desirable for solutions at high Strouhal number, which are necessary for construction of the transfer function. The axial derivatives are calculated using finite differences, and the spatial marching is performed using an implicit Euler method. A mapping function is used to transform the [-1 1] Chebyshev domain to an infinite one. Dirichlet boundary conditions are imposed on the flow variables at $r \rightarrow \infty$. This procedure should lead to reflected waves, ${ }^{12}$ but for a supersonic jet the reflected Mach waves should only influence downstream portions of the computational domain. ${ }^{13}$ Centerline conditions are dealt with using the procedure described by Mohseni \& Colonius. ${ }^{14}$

After spatial discretisation of the radial and axial directions and imposition of the boundary conditions, the system in (3) takes the form of the following downstream marching scheme:

$$
\left(\Delta x\left(A+B+D \mathcal{D}_{r}\right)+C\right)^{j+1} \hat{\mathbf{q}}^{j+1}=C^{j+1} \hat{\mathbf{q}}^{j},
$$

where $\mathcal{D}_{r}$ denotes the discrete radial derivative and spatial marching is realized to evolve the solution from $\Delta x_{j}$ to $\Delta x_{j+1}$. The minimum step-size derived by Li and Malik ${ }^{15}$

$$
\Delta x \geq \frac{1}{\operatorname{Re}\{\alpha\}},
$$

was employed in the code, for proper resolution of the acoustic field. ${ }^{5}$

The solution of this problem requires initial conditions for the shape functions $\hat{\mathbf{q}}$. These conditions are taken close to the nozzle exit, and are calculated by considering the parallel flow approximation $\frac{\partial \hat{\mathbf{q}}}{\partial x}=0$, which allows for the definition of a spatial stability eigenvalue problem,

$$
\left(A+B_{1}+D \frac{\partial}{\partial r}\right) \hat{\mathbf{q}}=\alpha\left(-B_{2}\right) \hat{\mathbf{q}}
$$

where the $B$ matrix is decomposed into $B_{1}+\alpha B_{2}$.

Since PSE is a linear method, the amplitude of the solution is undefined and it may be multiplied by a constant in order to fit the experimental data. For the transfer function approach, the division of the output solution by the input eliminates the need to determine the free constant.

Finally, temporal and azimuthal average of the numerical data of the Mach 1.5 isothermal supersonic jet obtained by the LES database of Brès et al. ${ }^{7}$ (LES case A1 for B118 conditions of the reference) was used to define the mean flow. The axial velocity was fitted with sums of Gaussian profiles, in order to avoid non-smoothness problems:

$$
\overline{u_{x}}=\left\{\begin{array}{l}
1 \text { if } r<R(x), \\
u_{e}(x)+\frac{1}{2}\left(u_{c}(x)-u_{e}(x)\right)\left(\exp \left[\frac{-(r-R(x))^{2}}{\delta_{1}^{2}(x)}\right]+\exp \left[\frac{-(r-R(x))^{2}}{\delta_{2}^{2}(x)}\right]\right), \text { otherwise, }
\end{array}\right.
$$

where the parameters $R(x), u_{c}(x), u_{e}(x), \delta_{1}(x)$ and $\delta_{2}(x)$ are determined from a least squares fit to the numerical data. A similar fit was taken for the temperature profile, given by

$$
\bar{T}=T_{e}(x)+T_{1}(x)\left(\exp \left[\frac{-\left(r-R_{T}(x)\right)^{2}}{\delta_{T 1}^{2}(x)}\right]+\exp \left[\frac{-\left(r+R_{T}(x)\right)^{2}}{\delta_{T 1}^{2}(x)}\right]\right)+T_{2}(x)\left(\exp \left[\frac{-(r)^{2}}{\delta_{T 2}^{2}(x)}\right]\right)
$$

where $T_{e}(x)$ is the temperature of the far field and the parameters $R_{T}(x), T_{1}(x), T_{2}(x), \delta_{T 1}(x)$ and $\delta_{T 2}(x)$ are also determined from a least squares fit to the numerical data. Once the temperature was modeled, density was obtained with the ideal gas law, considering constant pressure. Radial and azimuthal velocities were defined as zero.

\section{SISO Modelling Using PSE}

In this section, the linearity of PSE is exploited to obtain a SISO (single-input single-output) model between any two separated positions in a turbulent jet. 
Defining $R(\omega)$ and $Y(\omega)$ as the complex pressures at two axially and radially separated positions along the jet - where $\omega$ denotes the angular frequency - and assuming a linear relation between these, a frequency domain transfer function can be obtained as, ${ }^{16}$

$$
G(\omega)=\frac{Y(\omega)}{R(\omega)} .
$$

A time-domain transfer function, $g(t)$, is then available following an inverse Fourier transform,

$$
g(t)=\frac{1}{2 \pi} \int_{-\infty}^{\infty} G(\omega) e^{-i \omega t} d \omega .
$$

Once $g(t)$ is obtained, the output $y(t)$ of the system can be predicted by convolution with the input $r(t)$ and a SISO system is defined:

$$
y(t)=\int_{0}^{\infty} g(\tau) r(t-\tau) \mathrm{d} \tau .
$$

Equation 12 is only useful for real-time prediction of the flow behaviour when there is a causal relationship between input and output signals, $g(t<0)=0$. This is the case for convection dominated flows in which structures detected at a given position will evolve convectively, affecting downstream stations at later times.

PSE was thus used to calculate pressure fluctuations for the axisymmetric mode. The computation is repeated for many Strouhal numbers (the low computational cost of PSE makes this feasible), and a change of variables is necessary in order to perform the inverse Fourier transform:

$$
d \omega=2 \pi \frac{U_{j e t}}{D} d S t
$$

where $U_{j e t}$ is the jet velocity. Using this definition the inverse transform is,

$$
g(t)=2 \pi \frac{1}{2 \pi} \frac{U_{j e t}}{D} \int_{-\infty}^{\infty} G\left(\frac{U_{j e t}}{D} S t\right) e^{-i \frac{U_{j e t}}{D} S t . t} d S t .
$$

Or, by defining $F^{-1}[G(\omega)]$ as the Inverse Fourier Transform of $G(\omega)$ :

$$
g(t)=2 \pi \frac{U_{j e t}}{D} F^{-1}\left[G\left(\frac{U_{j e t}}{D} S t\right)\right] .
$$

Use of the scaling property of the Fourier Transform gives,

$$
g\left(t \frac{U_{j e t}}{D} 2 \pi\right)=F^{-1}[G(S t)] .
$$

Defining the non-dimensional time, $t^{*}=t \frac{U_{j e t}}{D}$, we have,

$$
g\left(2 \pi t^{*}\right)=F^{-1}[G(S t)] .
$$

\section{B. Empirical Transfer Function}

Based on pressure data of the LES of the Mach $1.5\left(M_{j}=1.5\right)$ isothermal ideally-expanded jet of Brès et al. ${ }^{7}$ using a procedure similar to that outlined in section 2, by assuming a linear relation between the pressure at two different axial locations along the jet, a frequency domain transfer function may be obtained as,

$$
G(\omega)=\frac{S_{r y}}{S_{r r}}
$$

where $S_{r r}$ and $S_{r y}$ are, respectively, the auto and cross-spectra of the input and output signals.

In order to improve the results, the initial period of the output signal and the final period of the input signal were discarded, similarly to what was done by Jaunet et al. ${ }^{17}$ The discarded period was taken as the lag of the peak cross-correlation of the unmodified input and output signals.

Inverse Fourier transform (equation 11) of this provides an empirical, time-domain transfer function that can be used to estimate downstream evolution of the pressure field based on upstream measurements (equation 12). The transfer function in the time-domain and the pressure estimates were later corrected to account the discarded period. 


\section{Results \& Discussion}

\section{A. Sample predictions using transfer functions}

The real part of the pressure obtained by PSE is shown in Figure 1 for a sample of Strouhal numbers. It can be seen that PSE is able to capture some of the pressure waves of the near acoustic field, consistently with previous results, ${ }^{5}$ which used the same database (LES case A1 for B118 conditions, ${ }^{7}$ which corresponds to a Mach 1.5 isothermal ideally-expanded jet).
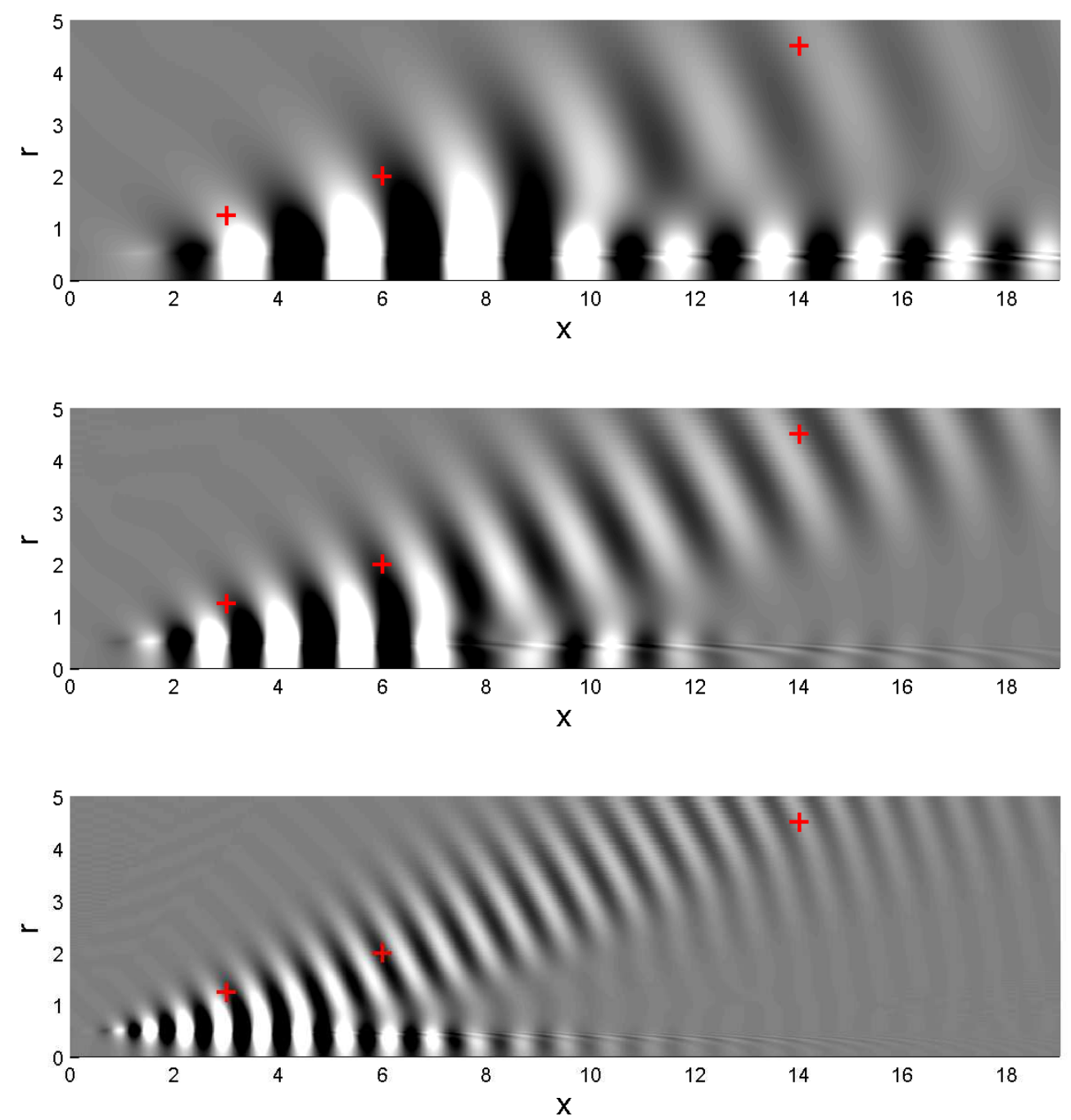

Figure 1. Pressure fluctuations for axisymmetric mode and Strouhal numbers equal to $0.3,0.5$ and 1.0 , respectively. Crosses indicate input and output positions of Figures 2, 3 and 4.

PSE was used to calculate the transfer function between pressures at different positions. As an example, Figures 2 and 3 show the transfer functions relating the pressure at input position $(x, r)=(3.0,1.25)$ and output positions $(x, r)=(6.0,2.0)$ and $(x, r)=(14.0,4.51)$. The first output position is a point in the near pressure field of the jet, whereas the second output is in the acoustic field, as the observation of the sample results in Figure 1 suggests. For both cases, there is a good agreement between empirical and PSE-based transfer functions, both in frequency (Figure 2) and in time domain (Figure 3). This is a first indication of the validity of the approaches for the present problem.

Using the unsteady pressure data of the LES database at $(x, r)=(3.0,1.25)$, we estimated the pressures at $(x, r)=(6.0,2.0)$ and $(x, r)=(14.0,4.51)$, the same outputs of Figures 2 and 3, using PSE and empirical transfer functions. We highlight that transfer functions were obtained for the axisymmetric mode, and all 


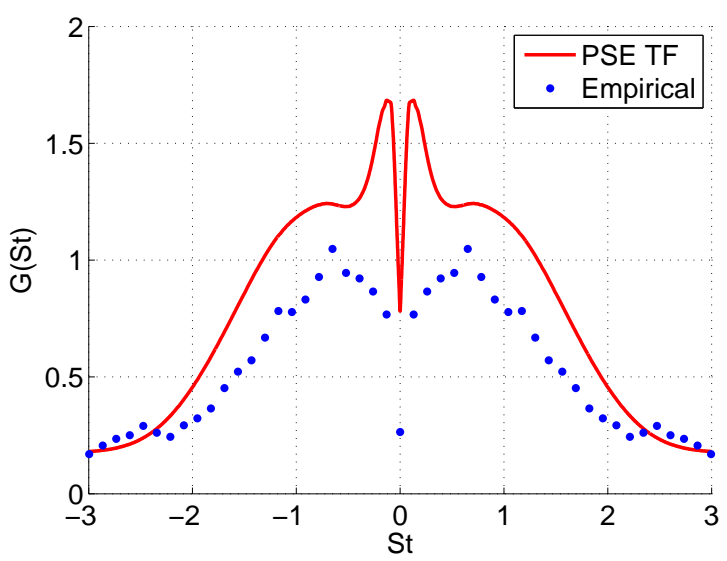

(a)

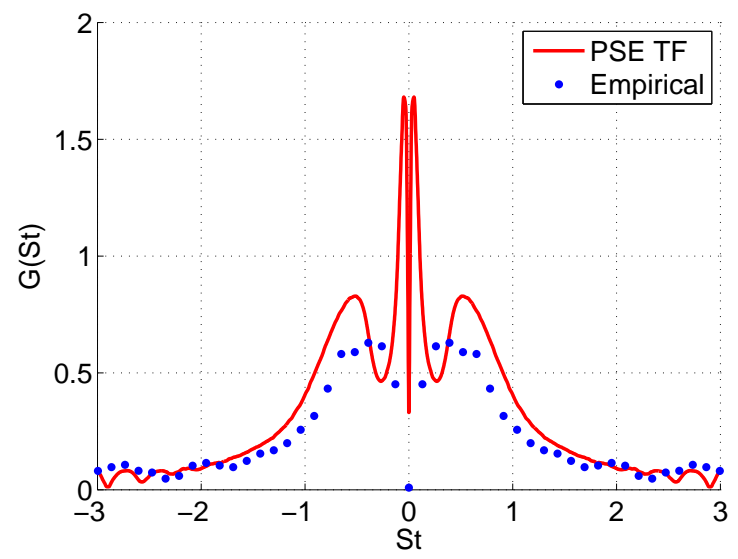

(b)

Figure 2. Transfer function in frequency-domain. (a) Input $(x, r)=(3.0,1.25)$ and output $(x, r)=(6.0,2.0)$. (b) Input $(x, r)=(3.0,1.25)$ and output $(x, r)=(14.0,4.51)$.

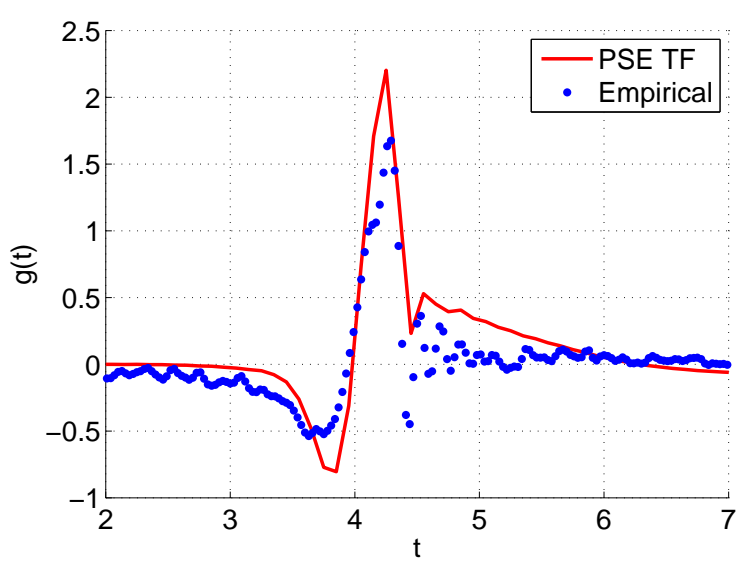

(a)

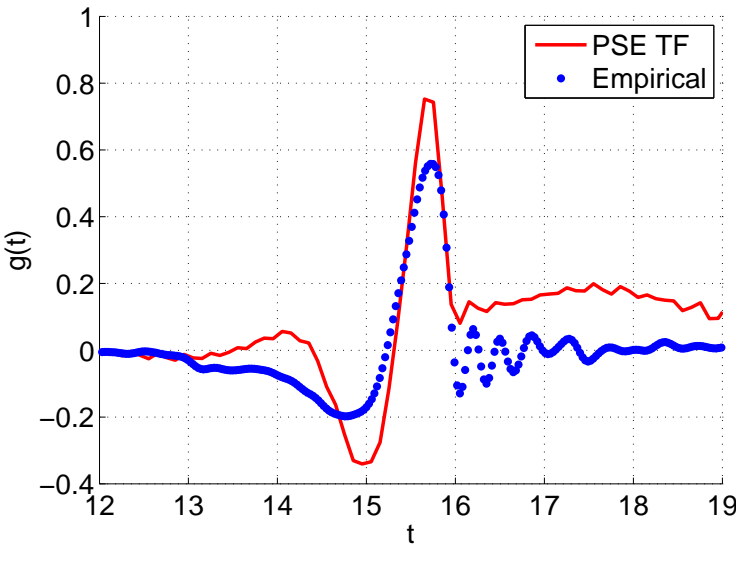

(b)

Figure 3. Transfer function in time-domain. (a) Input $(x, r)=(3 \cdot 0,1.25)$ and output $(x, r)=(6 \cdot 0,2.0)$. (a) Input $(x, r)=(3.0,1.25)$ and output $(x, r)=(14.0,4.51)$.

predictions in this work refer solely to this mode; accordingly, the inputs and outputs correspond to the axisymmetric part of the pressure at a given location. As can be seen in Figure 4, both PSE and empirical transfer functions show a reasonable agreement in estimating the axisymmetric part of the pressure at the output.

As expected, ${ }^{18}$ the agreement is lower for the most distant output, $(x, r)=(14.0,4.51)$. However, it is noteworthy that this linearized model could predict the pressure of a turbulent jet more than 11 diameters away from the position taken as input. Furthermore, a single near-field pressure input allowed a reasonable time-domain prediction of the radiated sound. This extends the results of Sinha et al. ${ }^{5}$ to the time domain, and suggests that the present transfer functions could be used for closed-loop control aiming directly at the reduction of Mach-wave radiation by supersonic jets.

\section{B. Parametric evaluation of transfer-function performance}

The agreement of the prediction was evaluated using the normalized cross-correlation of the output signals predicted by the transfer function and the LES results. A cross-correlation equals to unity shows that a given transfer function leads to predictions that perfectly represent the output time series, at least in shape, whereas a cross-correlation equals to zero implies that the prediction is orthogonal to the LES results, and thus completely inaccurate. 
As reference, the values of correlation were compared to the peak value of the magnitude-squared coherence estimate calculated using the LES pressure data at the input and output positions. It is expected that "jitter" in wavepacket behaviour would lead to coherence decay, ${ }^{19}$ which is not accounted for by either transfer function. We expect that transfer functions, which are based on a linearity assumption, could only lead to good predictions for a pair of input and output positions with high coherence.

A point near the boundary of the mixing layer $((x, r)=(4.0,1.0))$, a point in the near pressure field $((x, r)=(6.0,2.0))$ and a point in the acoustic field $((x, r)=(14.0,4.51))$ were chosen to be fixed as output positions. As can be seen in Figures 5, 7 and 9, the correlation obtained using PSE transfer functions can be considerable high if the input is, at least slightly, outside the mixing layer, whose approximate boundaries are represented with black lines. These results suggest that the near-field pressure measurements used by Sasaki et al. ${ }^{18}$ were thus taken at a favorable position for estimations based on transfer functions. The near-field pressure tends to be dominated by the signature of Kelvin-Helmholtz wavepackets, ${ }^{1,21-23}$ and is thus a more favorable position to use as an input to predict downstream behaviour, or, for supersonic jets, Mach-wave radiation (Figure 9).

Both inside and outside the mixing layer, the prediction using the empirical transfer functions has a higher correlation when compared to PSE transfer functions, which is expected, given the higher dependence on empirical data of this method. For inputs outside the mixing layer the correlation is comparable for both methods. However, for input points inside the mixing layer, these differences are more significant and PSE leads to worse predictions. Moreover, both methods show reduction of correlation inside the mixing layer. The lower values of peak coherence between input and output indicate that this lower correlation inside the mixing layer may be intrinsically related to the incoherent jitter in the turbulent field, which is not captured by the present linear models.

The ratio of the root mean square (RMS) of the pressure predicted by the transfer functions to the RMS of the pressure from the LES database is presented in Figures 6,8 and 10. This is relevant, since the normalized correlation only shows similarity in shape between the predicted time series and the reference LES results, hence, an accurate prediction should also match the RMS value of the output. While the PSE transfer functions may underestimate or overestimate the pressure flutuations, the empirical transfer function always underestimate the RMS, which is expected due to the method for estimating the function. ${ }^{24}$

The RMS ratio also show that PSE predictions have better agreement if the input position is outside the mixing layer. Inside the mixing layer the ratio of the RMS is close to 1.0 only in a small region (between $x=2$ and $x=4)$. This is a region where the Kelvin-Helmholtz wavepackets tend to dominate the dynamics, and this dominance may be relevant to extract pressure signals allowing the prediction of downstream behaviour using transfer functions.

To further investigate this issue, the peak cross-correlation was ploted for two input positions, one inside the mixing layer $((x, r)=(3.0,0.51))$ and one outside the mixing layer $((x, r)=(3.0,1.0))$, for a longitudinal position $x=3$ where good agreement of RMS has been identified. It can be noted in Figures 11 and 12 that the prediction capability of PSE is greatly reduced inside the mixing layer. We could not accurately predict the pressure if the output is a few diameters of distance when the input is inside the mixing layer. It is noticeable that there is a sharp decay of coherence between the input in the mixing layer and the downstream points, which is consistent with the worse performance of transfer function predictions.

The agreement is much better if both input and output are outside the mixing layer, which is consistent with Figures 5, 7 and 9. RMS results (Figures 13 and 14) also show better agreement if the input position is outside the mixing layer, an agreement that extends into the acoustic field.

\section{Conclusions}

A model based on the Parabolised Stability Equations was applied to an isothermal Mach 1.5 supersonic jet in order to construct time-domain transfer functions that can be used to estimate the unsteady pressure fluctuations, given the measureament of an upstream input. These transfer functions were compared to empirical functions obtained using results from a database of a well-validated large-eddy simulation of the jet. The prediction of pressure was compared to the results of the LES and showed good agreement, especially if the input position is, at least slightly, outside the mixing layer. For this region, pressure estimates using the PSE transfer functions are comparable to the results of empirical transfer functions. Such trend had already been observed for a subsonic jet and further demonstrates the pertinence of wavepacket models for applications in turbulent jets. ${ }^{18}$ 
A particularity of supersonic jets is that wavepacket models allow prediction of Mach-wave radiation..$^{5,25}$ Accordingly, with the transfer functions applied to the Mach 1.5 jet it is possible to predict, with good accuracy, the time series of pressure fluctuations in the acoustic field.

It has been shown that the PSE prediction, evaluated quantitatively by considering the correlation coefficient between prediction and LES time series, is deteriorated if the input position is inside the mixing layer. On the other hand, the method using empirical transfer functions shows better correlation in this region. Worse agreements are shown to arise where there is substantial coherence decay between input and output points, which indicates that dynamics cannot be accurately modeled using a single-input-single-output linear model.

The PSE-based approach has the added advantage of requiring only the mean flow to the construction of the transfer function, without the need of unsteady data of the flow field. Additionally, since usually microphones are allocated outside the mixing layer, the worse predictions of the method for input positions inside the mixing layer may not be a strong limitation in an experimental configuration, particularly for aeroacoustic applications. In this sense, similar positions were taken by Sasaki et al. ${ }^{18}$

Finally, given that, for the supersonic case, PSE has been show to agree with reasonable accuracy with the actual radiated acoustic emissions, the extension of this method to time-domain predictions is of practical interest. This feature indicates that it may be possible to use similar transfer functions for closed-loop control targeting directly the acoustic field radiated from a supersonic jet, also supplying means to determine the most appropriate positions for sensor/actuator placement.

\section{Acknowledgments}

This work has been funded by CNPq (grant number 444796/2014-2). A. Cavalieri was supported by a $\mathrm{CNPq}$ research scholarship. Kenzo Sasaki acknowledges the financial support from Capes via a $\mathrm{PhD}$ scholarship.

\section{References}

${ }^{1}$ Gudmundsson, K. and Colonius, T., "Instability wave models for the near-field fluctuations of turbulent jets," Journal of Fluid Mechanics, Vol. 689, 2011, pp. 97-128.

${ }^{2}$ Cavalieri, A. V., Rodríguez, D., Jordan, P., Colonius, T., and Gervais, Y., "Wavepackets in the velocity field of turbulent jets," Journal of fluid mechanics, Vol. 730, 2013, pp. 559-592.

${ }^{3}$ Sasaki, K., Piantanida, S., Cavalieri, A. V., and Jordan, P., "Real-time modelling of wavepackets in turbulent jets," 21th AIAA/CEAS Aeroacoustic Conference and Exhibit, 2015.

${ }^{4}$ Sasaki, K., Tissot, G., Cavalieri, A. V., Silvestre, F., Jordan, P., and Biau, D., "Closed-loop control of wavepackets in a free shear-flow," 22nd AIAA/CEAS Aeroacoustics Conference, 2016.

${ }^{5}$ Sinha, A., Rodríguez, D., Brès, G. A., and Colonius, T., "Wavepacket models for supersonic jet noise," Journal of Fluid Mechanics, Vol. 742, 2014, pp. 71-95.

${ }^{6}$ Brès, G. A., Nichols, J. W., Lele, S. K., and Ham, F. E., "Towards best practices for jet noise predictions with unstructured large eddy simulations," 42nd AIAA Fluid Dynamics Conference and Exhibit, 2012.

${ }^{7}$ Brès, G. A., Ham, F. E., Nichols, J. W., and Lele, S. K., "Unstructured Large-Eddy Simulations of Supersonic Jets," AIAA Journal, 2017.

${ }^{8}$ Crighton, D. and Gaster, M., "Stability of slowly diverging jet flow," Journal of Fluid Mechanics, Vol. 77, No. 02, 1976, pp. 397-413.

${ }^{9}$ Herbert, T., "Parabolized stability equations," Annual Review of Fluid Mechanics, Vol. 29, No. 1, 1997, pp. $245-283$.

${ }^{10}$ Sasaki, K., Estudo e controle de pacotes de onda em jatos utilizando as equações de estabilidade parabolizadas (in Portuguese), Master's thesis, Instituto Tecnológico de Aeronáutica, 2014.

${ }^{11}$ Rodríguez, D., Cavalieri, A. V., Colonius, T., and Jordan, P., "A study of linear wavepacket models for subsonic turbulent jets using local eigenmode decomposition of PIV data," European Journal of Mechanics-B/Fluids, Vol. 49, 2015 , pp. 308-321.

${ }^{12}$ Colonius, T. and Lele, S. K., "Computational aeroacoustics: progress on nonlinear problems of sound generation," Progress in Aerospace sciences, Vol. 40, No. 6, 2004, pp. 345-416.

${ }^{13}$ Malik, M. and Chang, C.-L., "Nonparallel and nonlinear stability of supersonic jet flow," Computers E fluids, Vol. 29, No. 3, 2000, pp. 327-365.

${ }^{14}$ Mohseni, K. and Colonius, T., "Numerical treatment of polar coordinate singularities," Journal of Computational Physics, Vol. 157, No. 2, 2000, pp. 787-795.

${ }^{15}$ Li, F. and Malik, M. R., "Spectral analysis of parabolized stability equations," Computers \& fluids, Vol. 26, No. 3, 1997, pp. 279-297. 2011.

${ }^{16}$ Bendat, J. S. and Piersol, A. G., Random data: analysis and measurement procedures, Vol. 729, John Wiley \& Sons, 
${ }^{17}$ Jaunet, V., Jordan, P., and Cavalieri, A., "Two-point coherence of wave packets in turbulent jets," Physical Review Fluids, Vol. 2, No. 2, 2017, pp. 024604.

${ }^{18}$ Sasaki, K., Piantanida, S., Cavalieri, V. G., and Jordan, P., "Real-time modelling of wavepackets in turbulent jets," Journal of Fluid Mechanics, 2017, In press.

${ }^{19}$ Cavalieri, A. V. and Agarwal, A., "Coherence decay and its impact on sound radiation by wavepackets," Journal of Fluid Mechanics, Vol. 748, 2014, pp. 399-415.

${ }^{20}$ Kœnig, M., Sasaki, K., Cavalieri, A. V., Jordan, P., and Gervais, Y., "Jet-noise control by fluidic injection from a rotating plug: linear and nonlinear sound-source mechanisms," Journal of Fluid Mechanics, Vol. 788, 2016, pp. 358-380.

${ }^{21}$ Suzuki, T. and Colonius, T., "Instability waves in a subsonic round jet detected using a near-field phased microphone array," Journal of Fluid Mechanics, Vol. 565, 2006, pp. 197-226.

${ }^{22}$ Tinney, C. and Jordan, P., "The near pressure field of co-axial subsonic jets," Journal of Fluid Mechanics, Vol. 611, 2008, pp. 175-204.

${ }^{23}$ Breakey, D. E., Jordan, P., Cavalieri, A., and Léon, O., "Near-field wavepackets and the far-field sound of a subsonic jet," 19th AIAA/CEAS Aeroacoustics Conference, 2013, p. 2083.

${ }^{24}$ Rocklin, G. T., Crowley, J., and Vold, H., "A comparison of H1, H2, and Hv frequency response functions," Proceedings of the 3rd international Modal Analysis Conference, Vol. 1, 1985, pp. 272-278.

${ }^{25}$ Tam, C. K. and Burton, D. E., "Sound generated by instability waves of supersonic flows. Part 2. Axisymmetric jets," Journal of Fluid Mechanics, Vol. 138, 1984, pp. 273-295. 


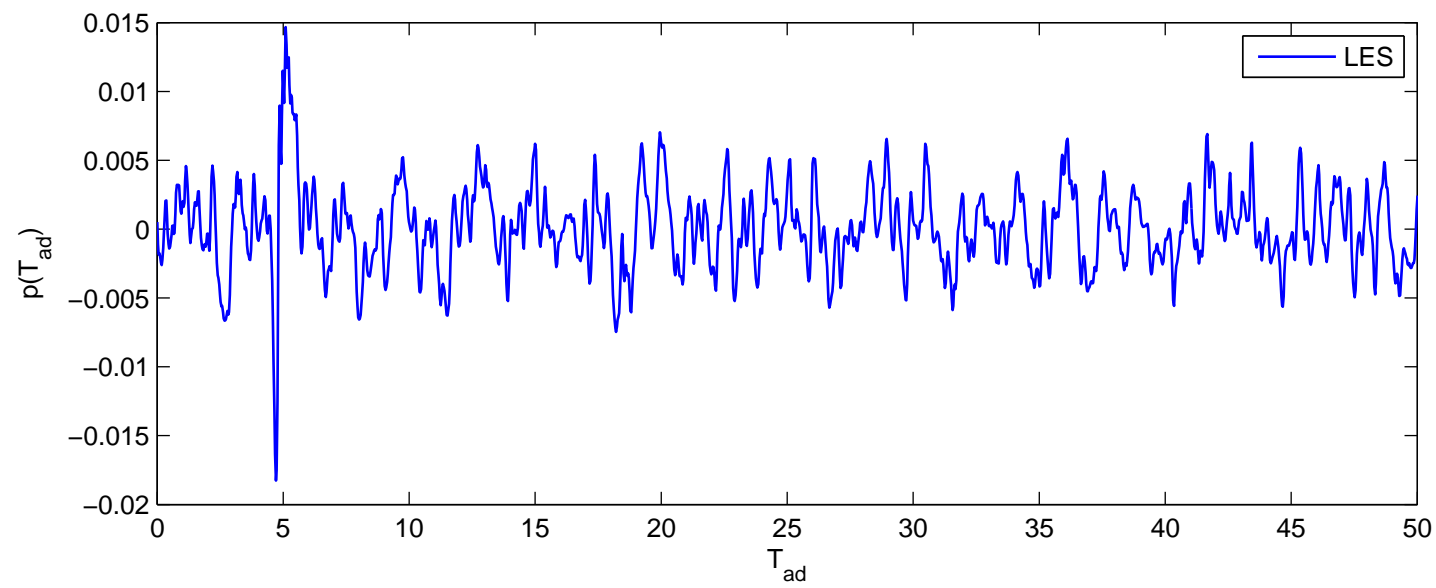

(a)

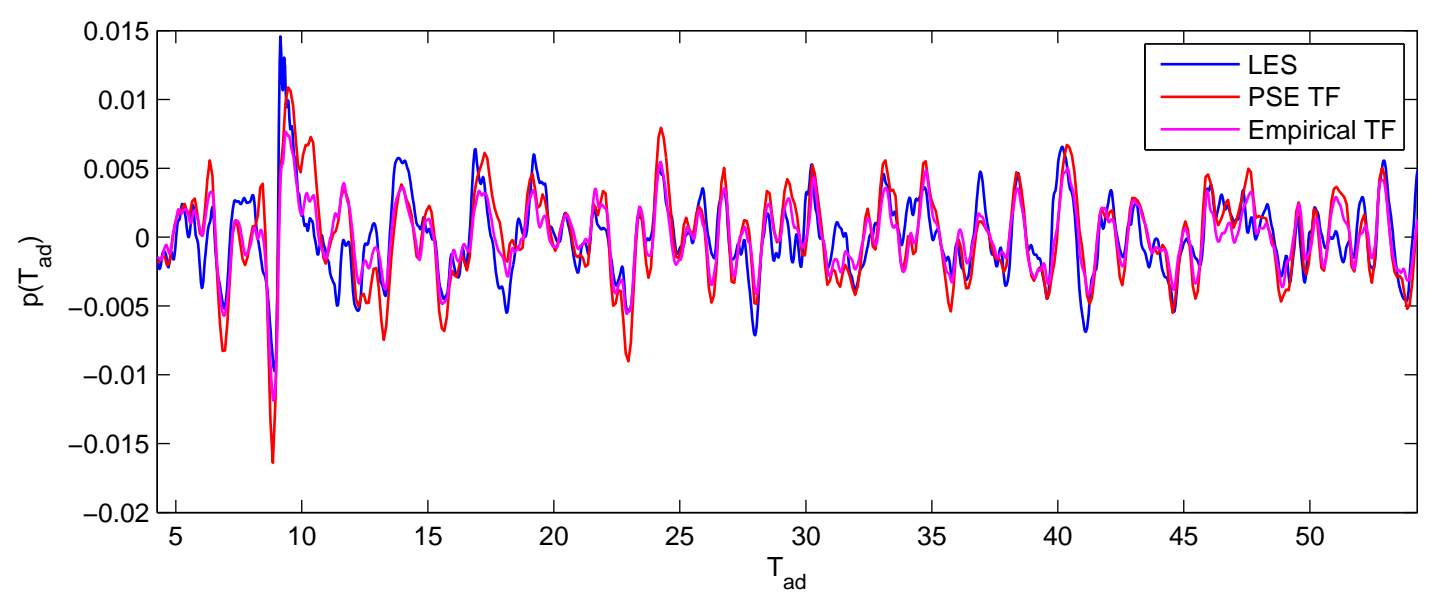

(b)

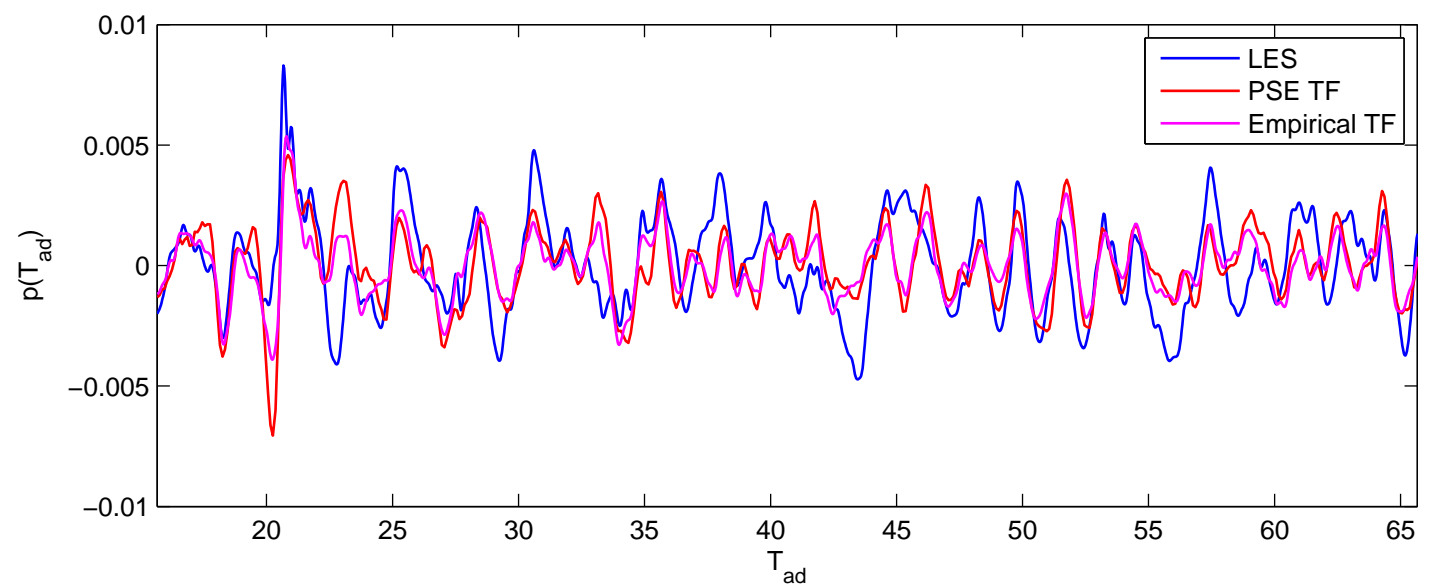

(c)

Figure 4. (a) Pressure at input $(x, r)=(3.0,1.25)$. (b) Pressure at $(x, r)=(6.0,2.0)$ predicted using the pressure at $(x, r)=(3.0,1.25)$. (c) Pressure at $(x, r)=(14.0,4.51)$ predicted using the pressure at $(x, r)=(3.0,1.25)$. 


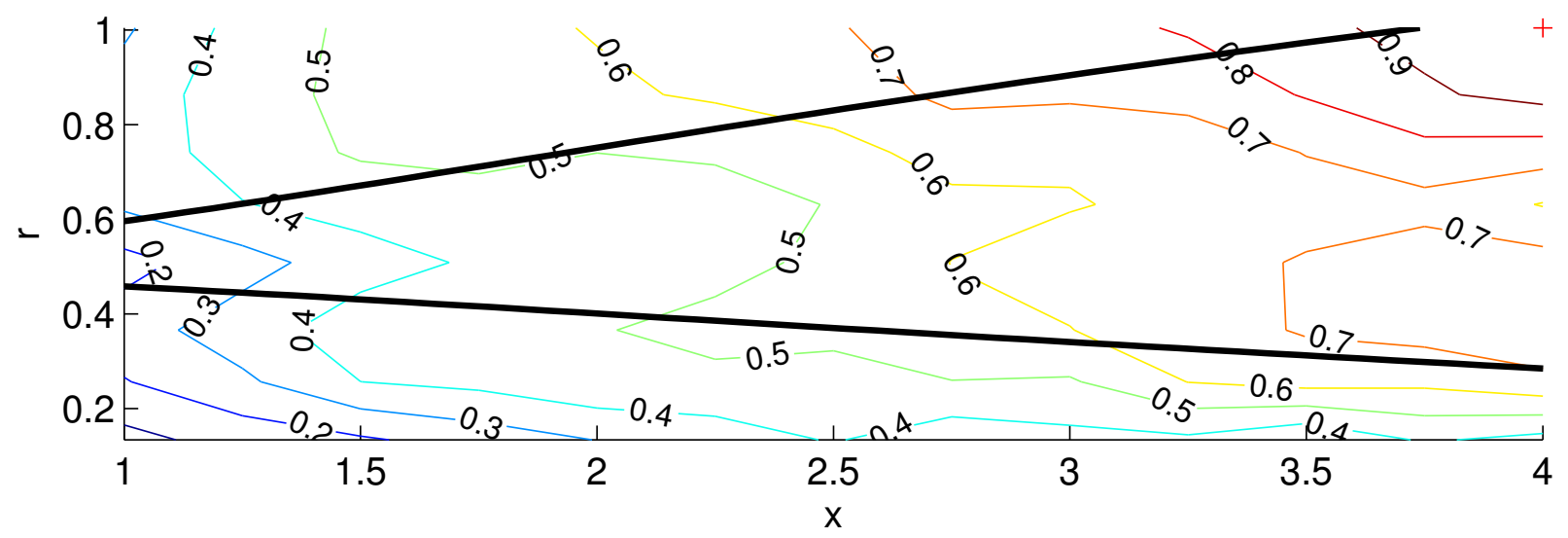

(a)

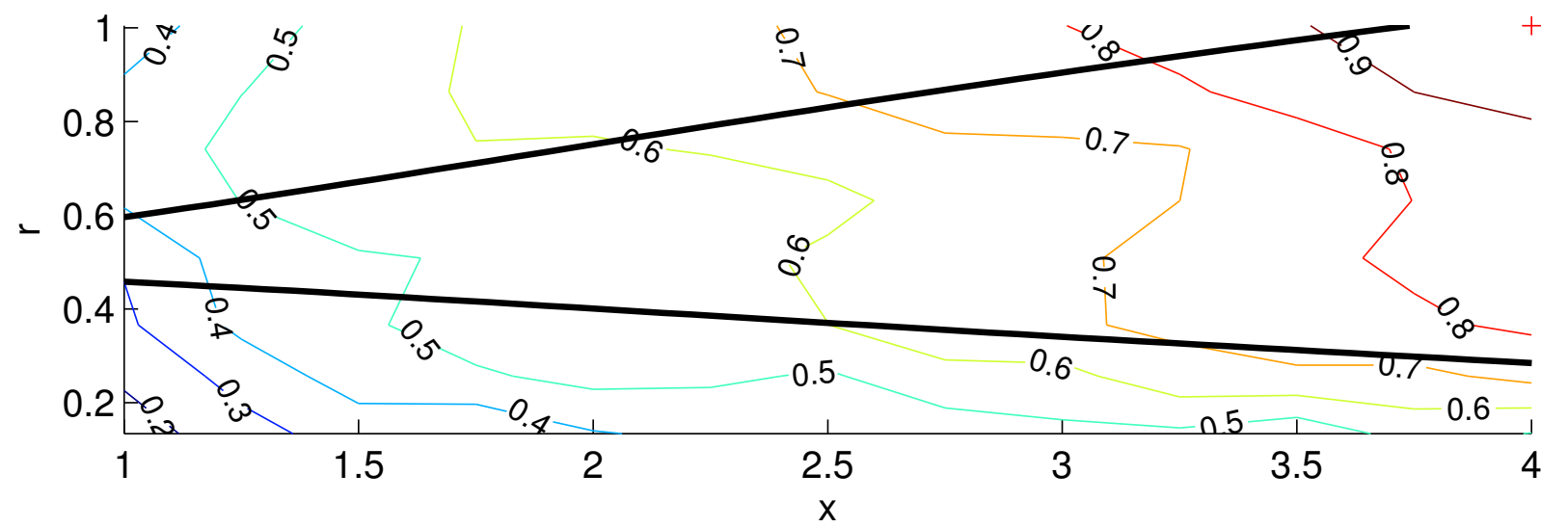

(b)

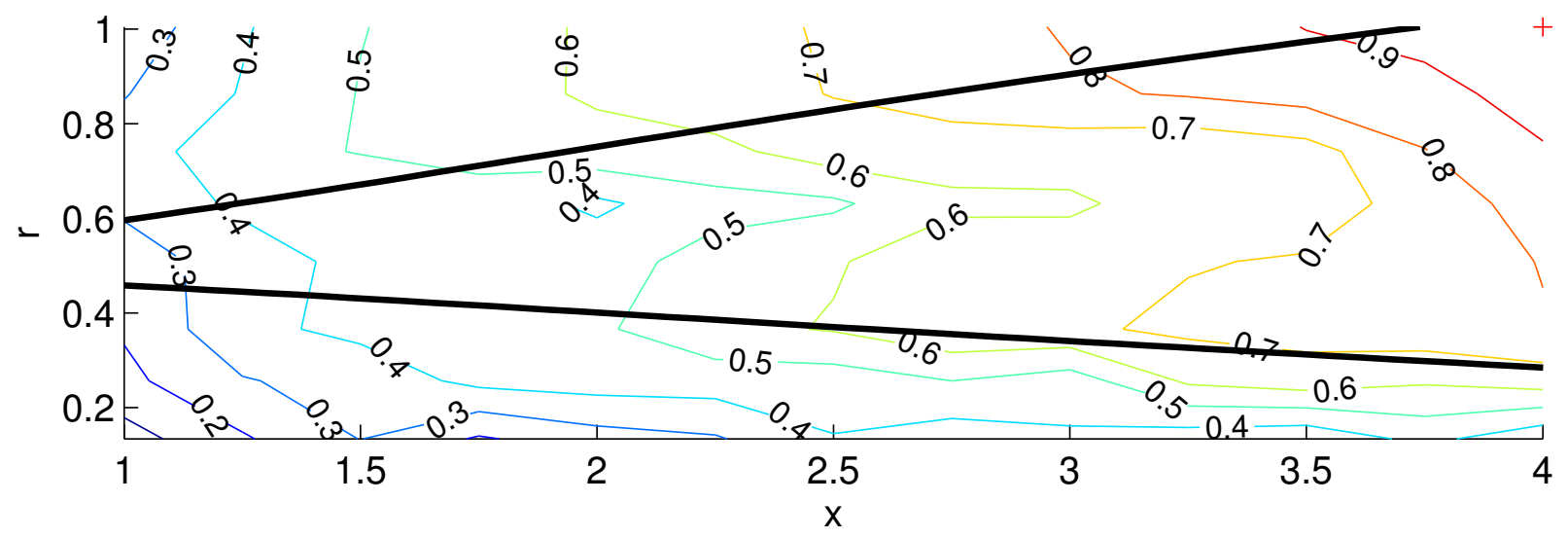

(c)

Figure 5. Contour of peak cross-correlation and peak coherence for several input positions. Output at $(x, r)=$ $(4.0,1.0)$ (indicated by cross). Black line indicates limits of mixing layer. (a) Peak cross-correlation using PSE transfer functions. (b) Peak cross-correlation using empirical transfer functions. (c) Peak coherence. 


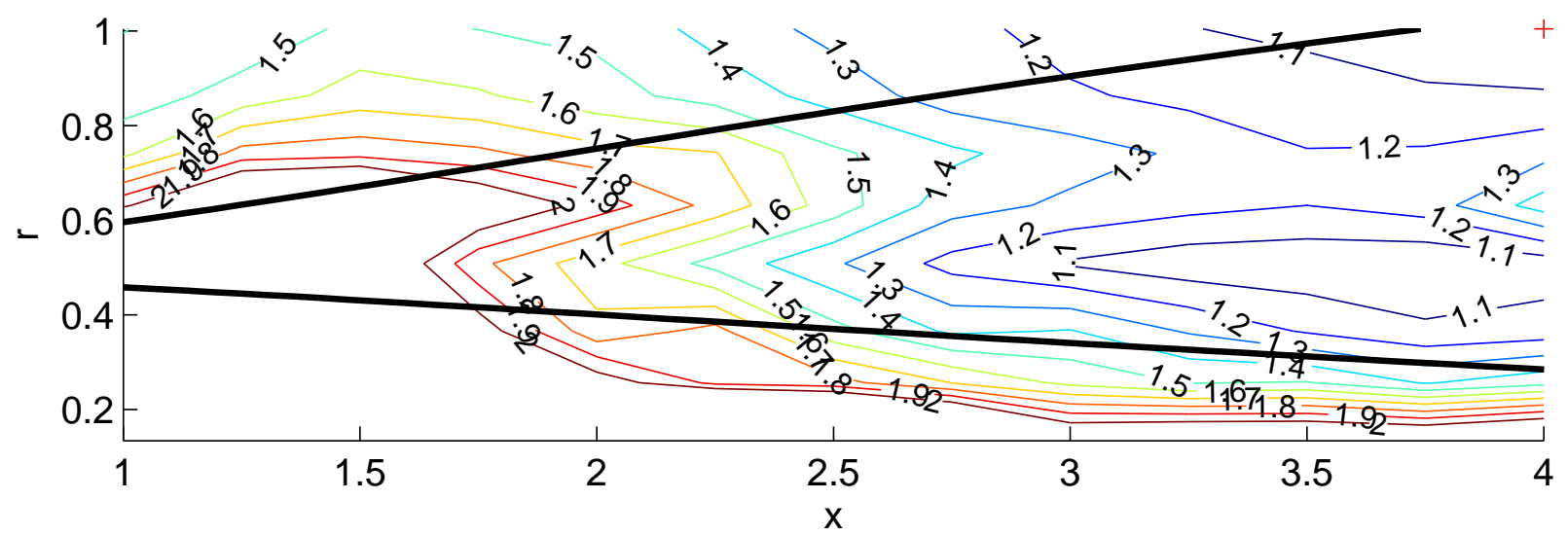

(a)

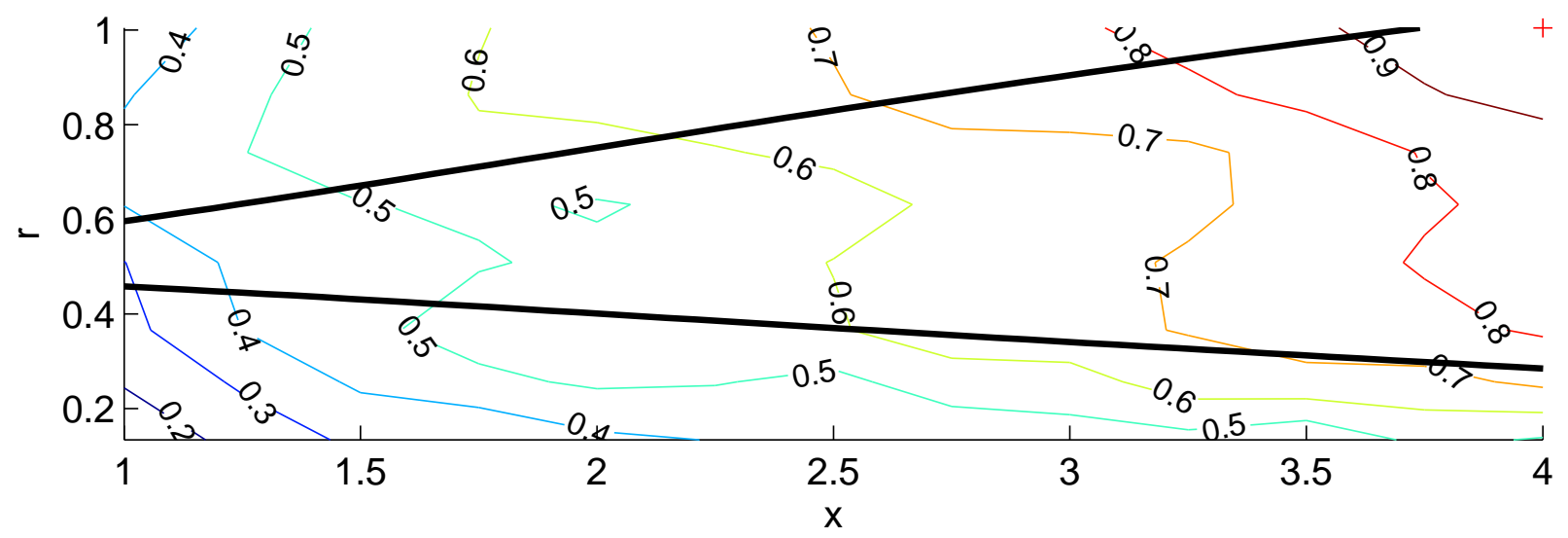

(b)

Figure 6. Ratio of root mean square (RMS) of pressure predicted by transfer function to RMS of pressure of LES. Output at $(x, r)=(4.0,1.0)$ (indicated by cross). Black line indicates limits of mixing layer. (a) RMS using PSE transfer functions. (b) RMS using empirical transfer functions. 


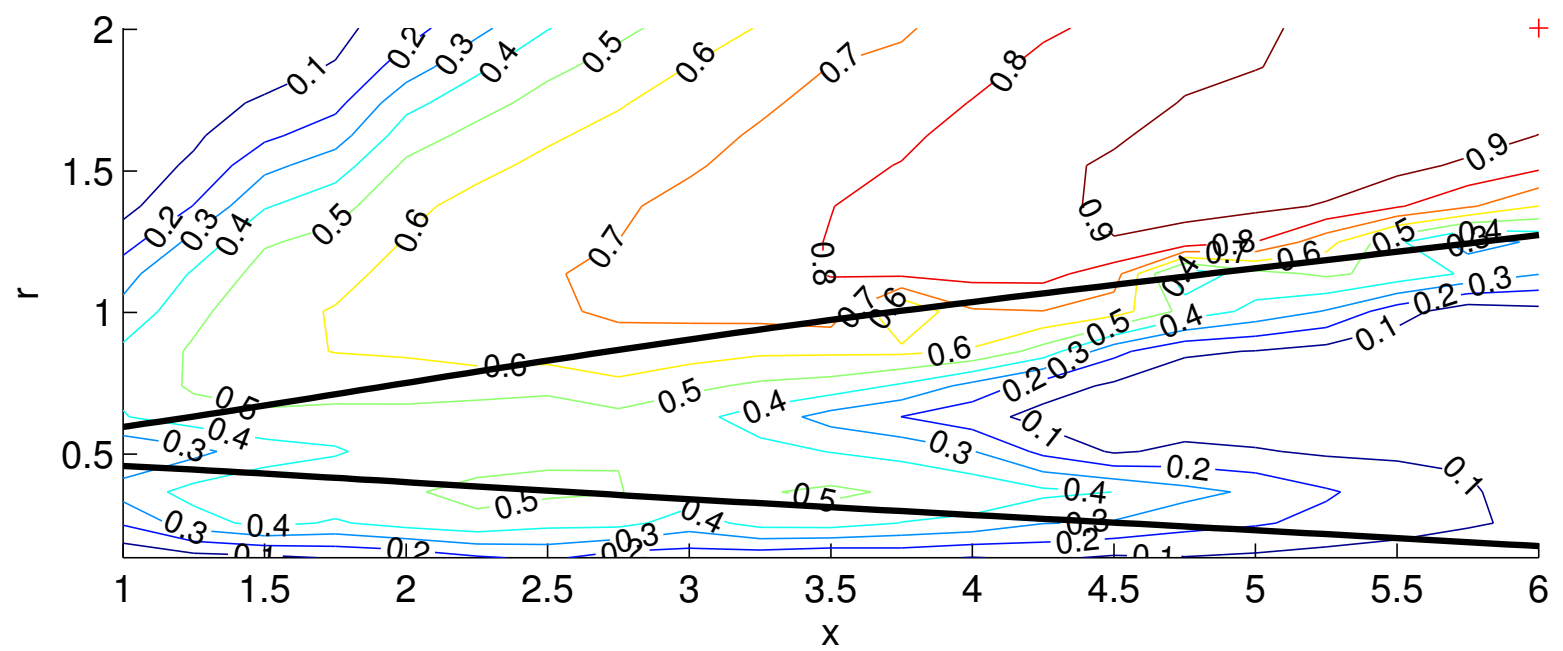

(a)

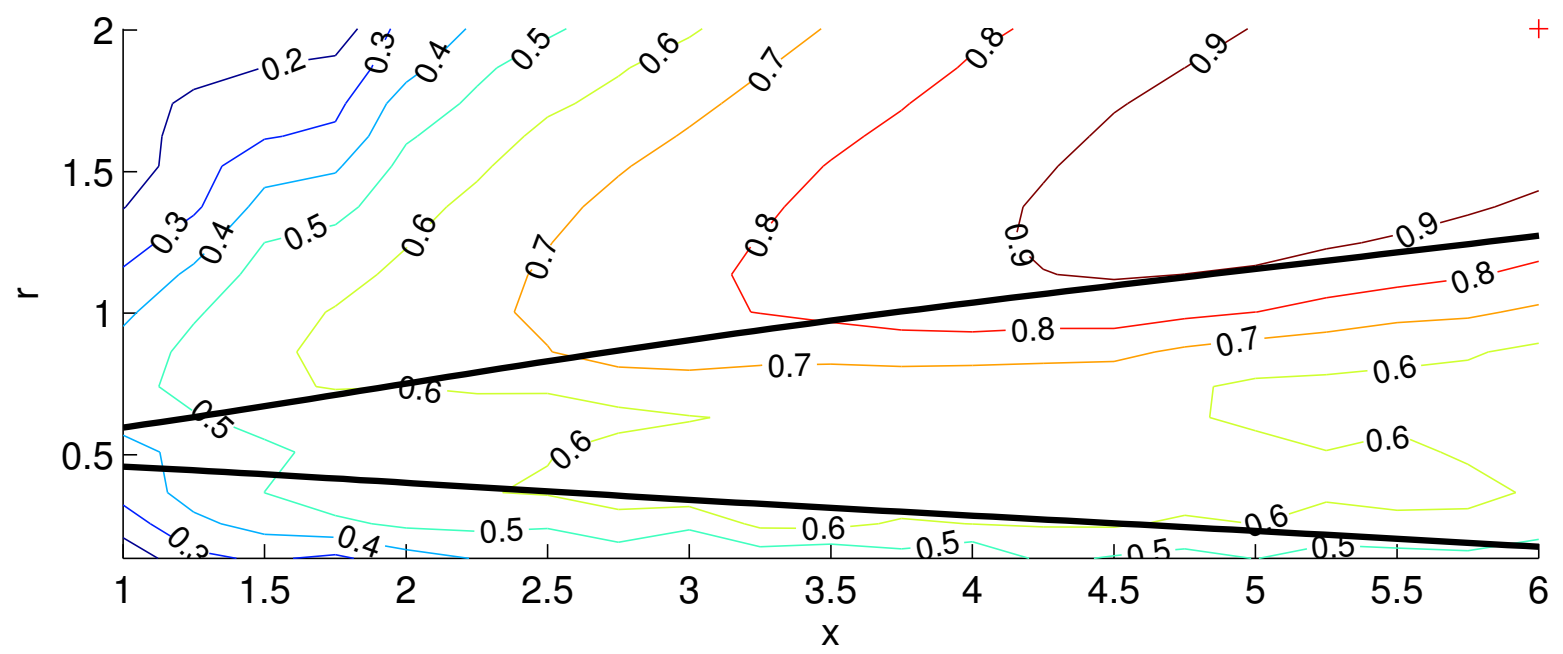

(b)

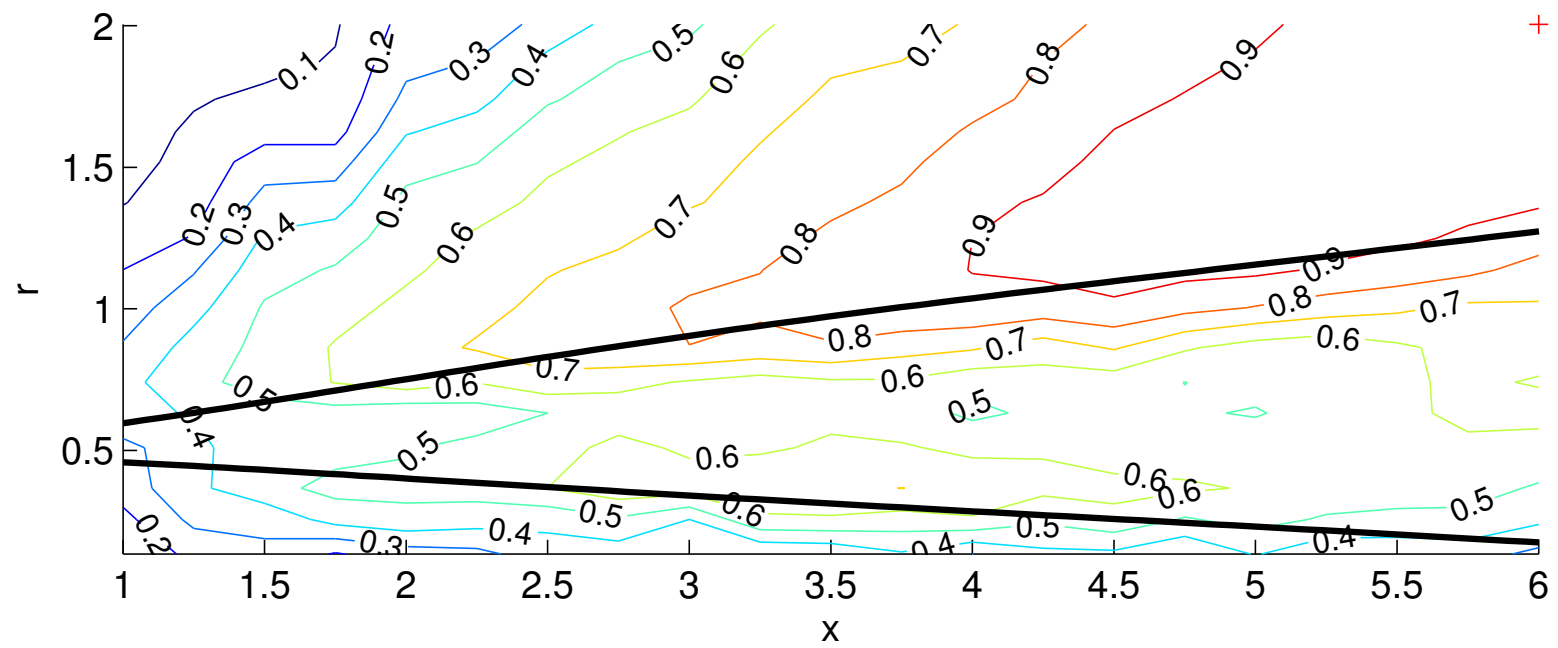

(c)

Figure 7. Contour of peak cross-correlation and peak coherence for several input positions. Output at $(x, r)=$ $(6.0,2.0)$ (indicated by cross). Black line indicates limits of mixing layer. (a) Peak cross-correlation using PSE transfer functions. (b) Peak cross-correlation using empirical transfer functions. (c) Peak coherence. 


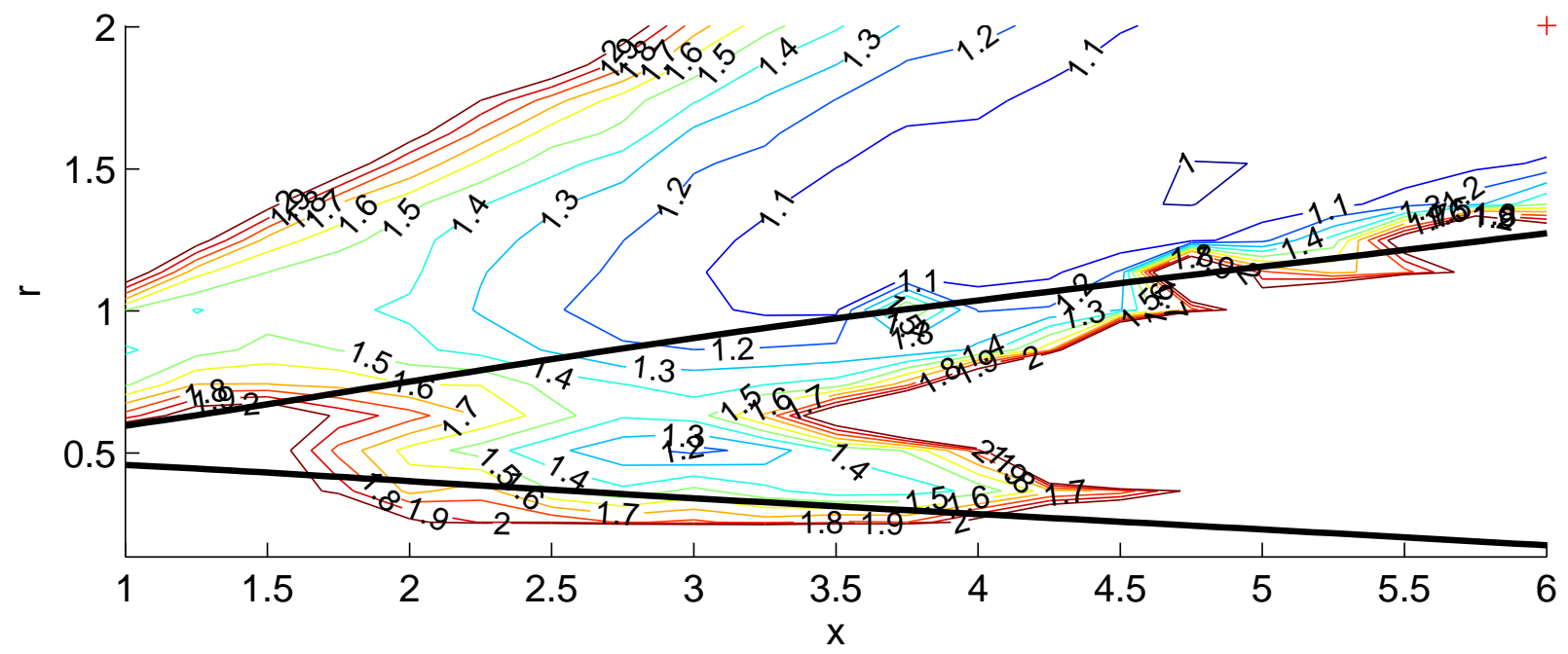

(a)

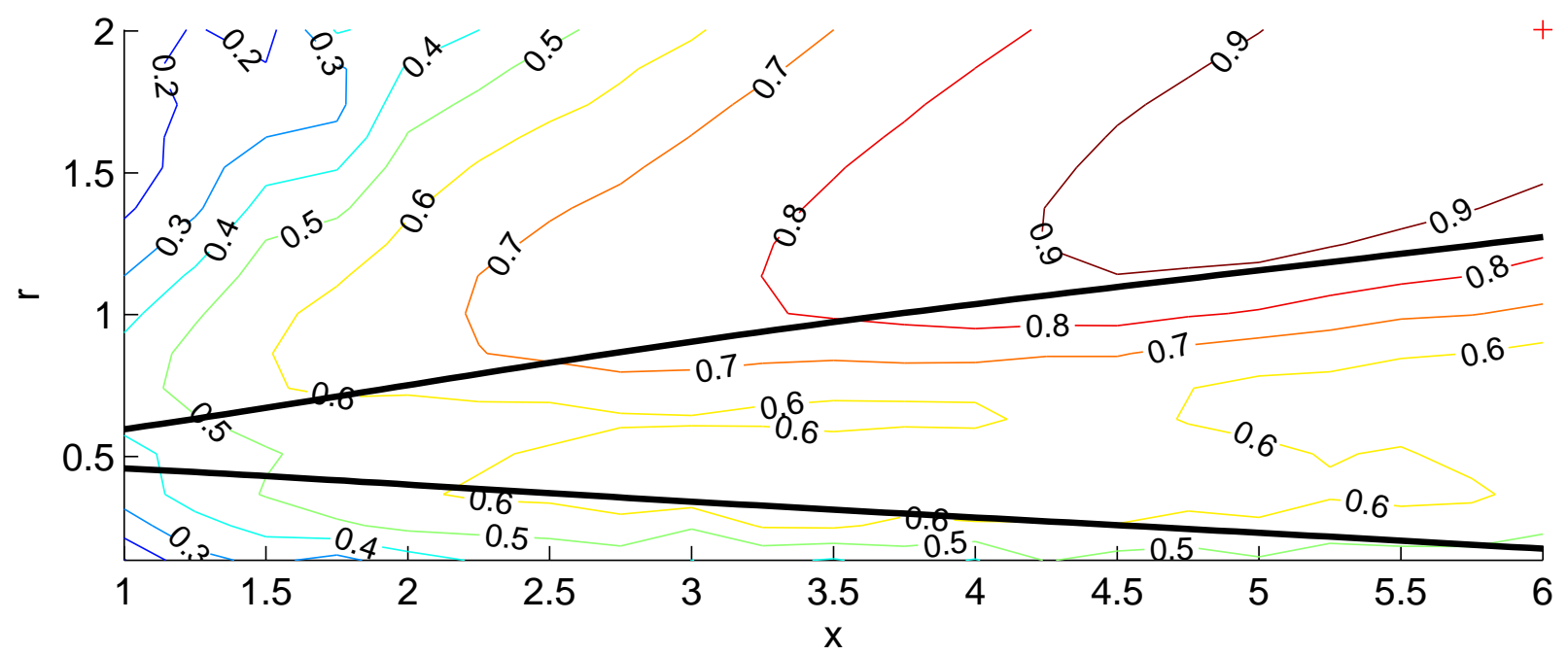

(b)

Figure 8. Ratio of root mean square (RMS) of pressure predicted by transfer function to RMS of pressure of LES. Output at $(x, r)=(6.0,2.0)$ (indicated by cross). Black line indicates limits of mixing layer. (a) RMS using PSE transfer functions. (b) RMS using empirical transfer functions. 


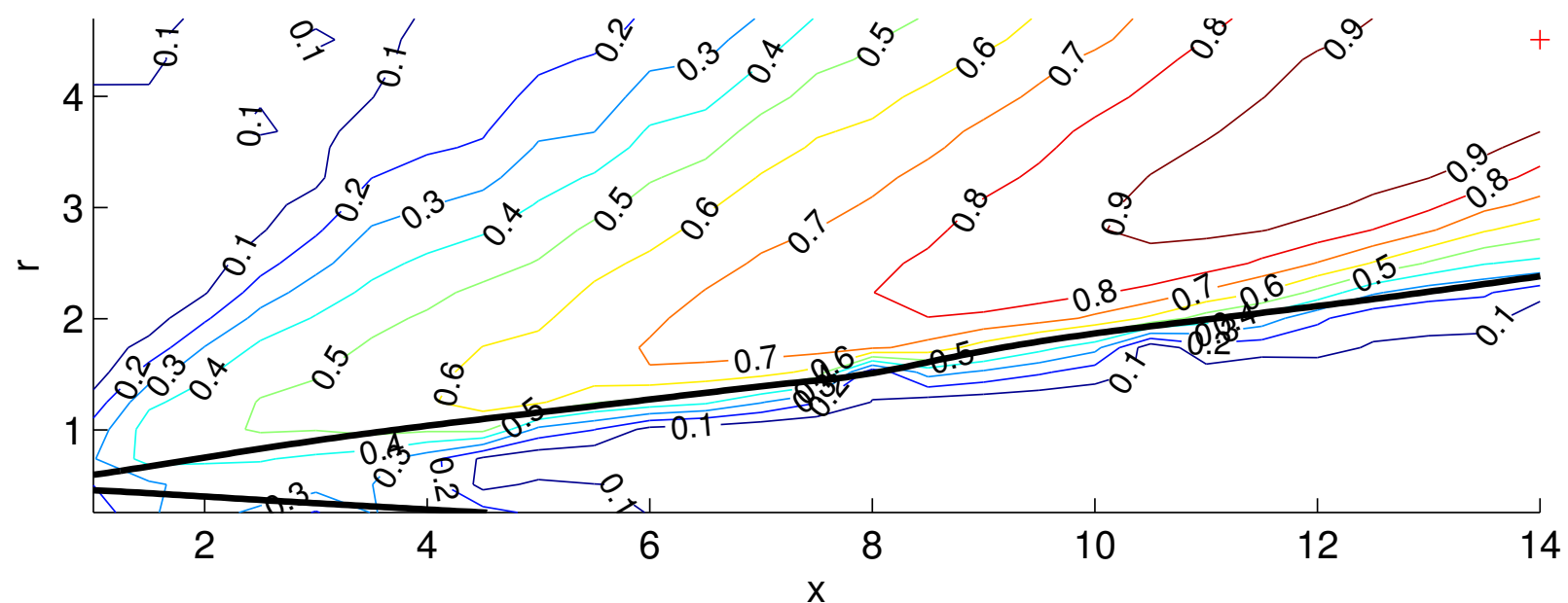

(a)

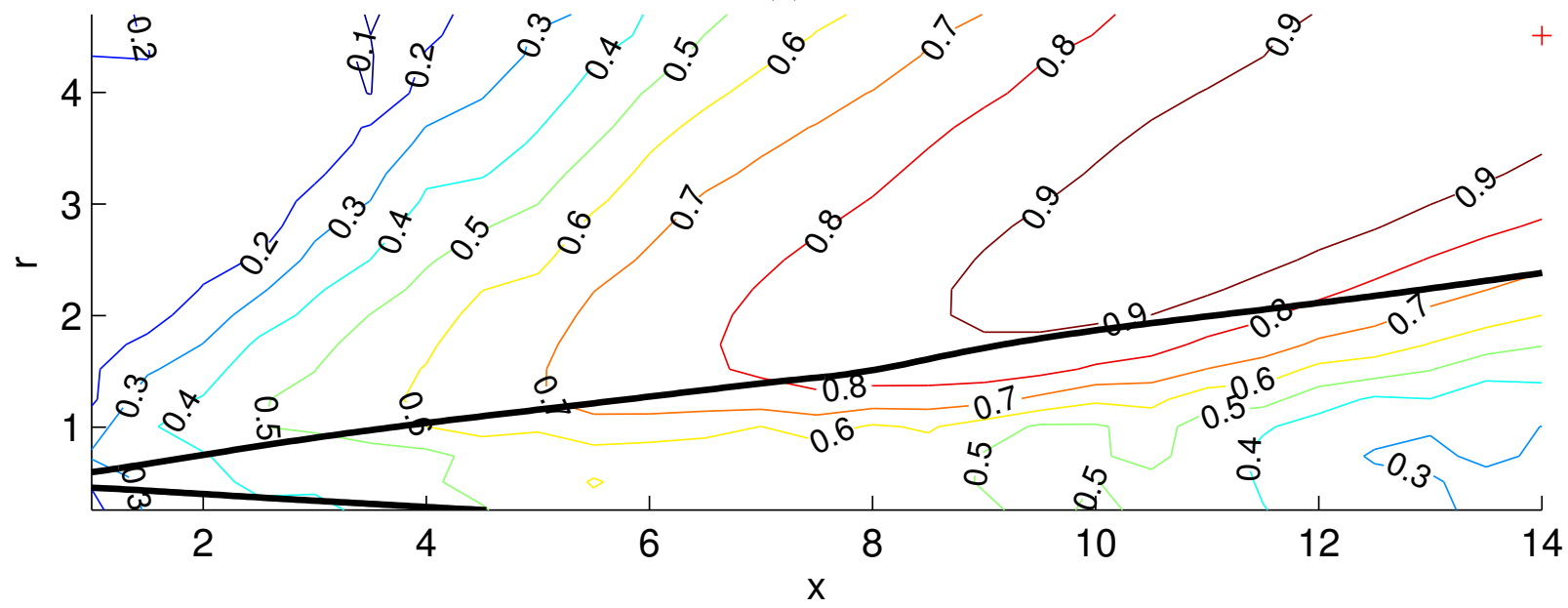

(b)

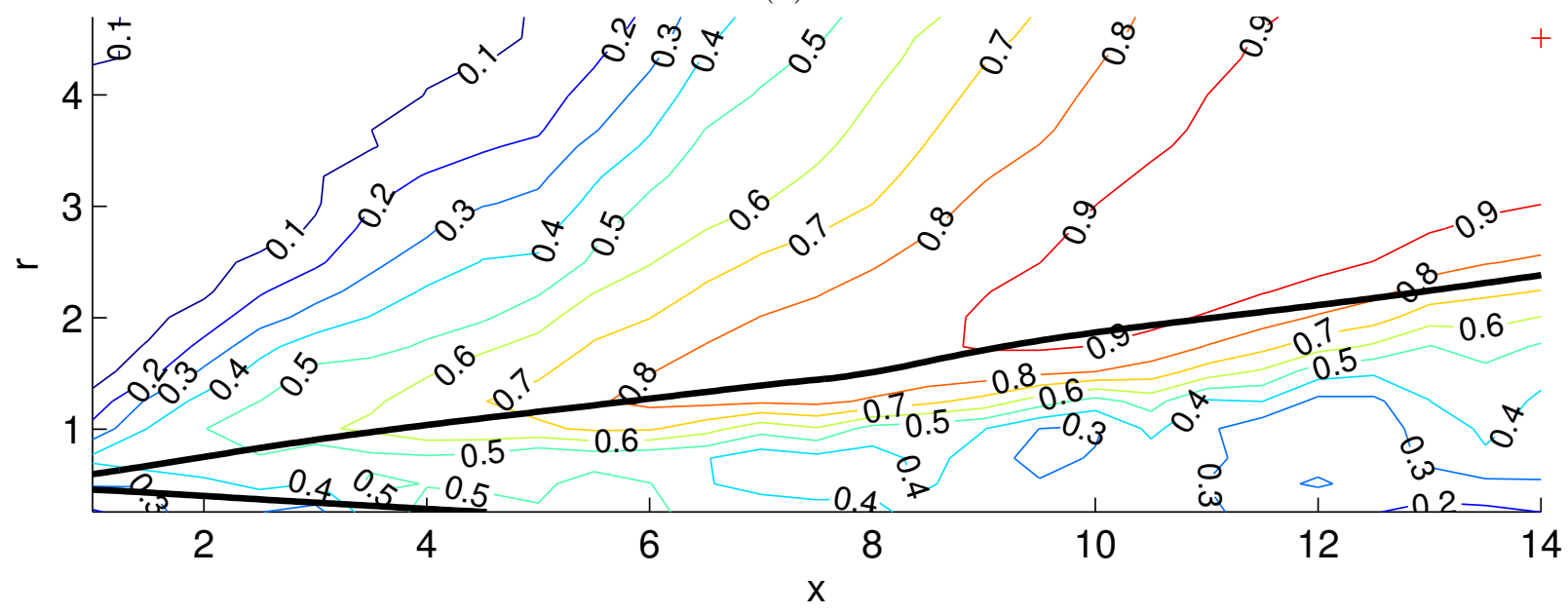

(c)

Figure 9. Contour of peak cross-correlation and peak coherence for several input positions. Output at $(x, r)=$ $(14.0,4.51)$ (indicated by cross). Black line indicates limits of mixing layer. (a) Peak cross-correlation using PSE transfer functions. (b) Peak cross-correlation using empirical transfer functions. (c) Peak coherence. 


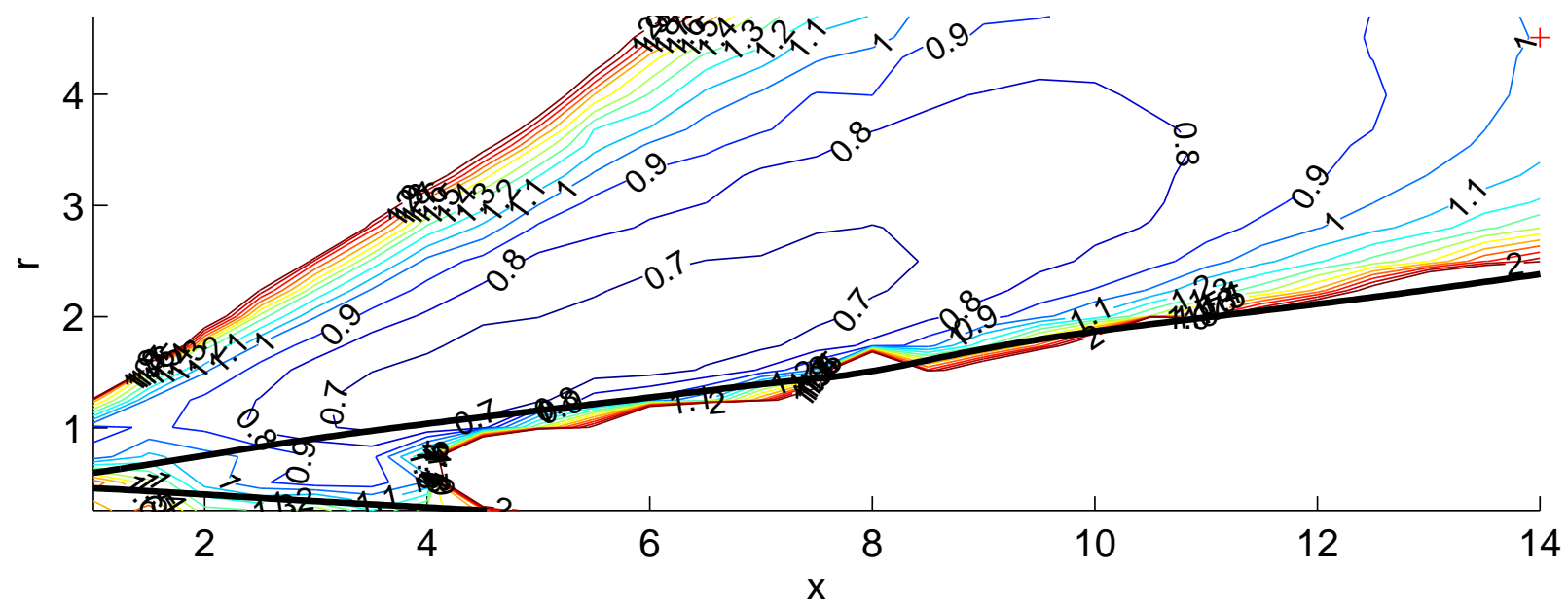

(a)

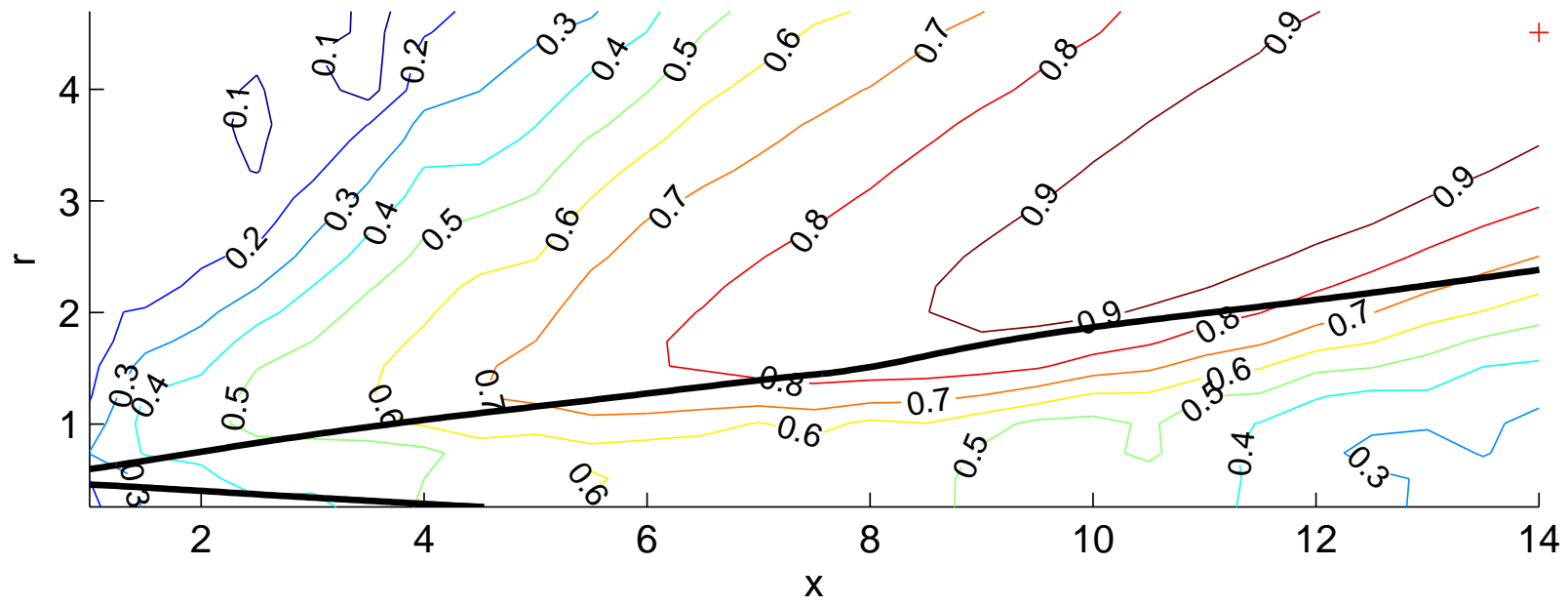

(b)

Figure 10. Ratio of root mean square (RMS) of pressure predicted by transfer function to RMS of pressure of LES. Output at $(x, r)=(14.0,4.51)$ (indicated by cross). Black line indicates limits of mixing layer. (a) RMS using PSE transfer functions. (b) RMS using empirical transfer functions. 


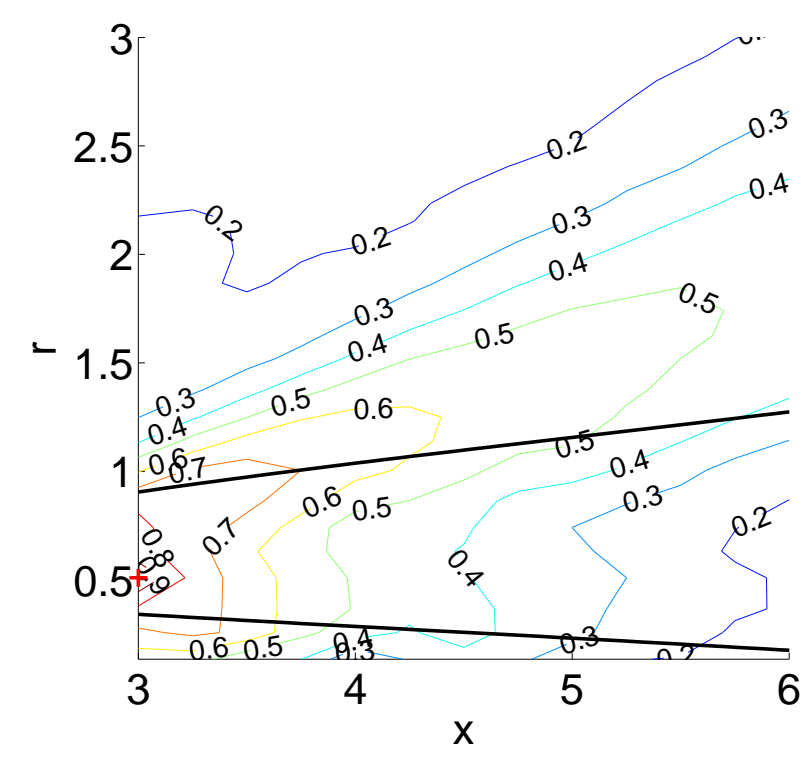

(a)

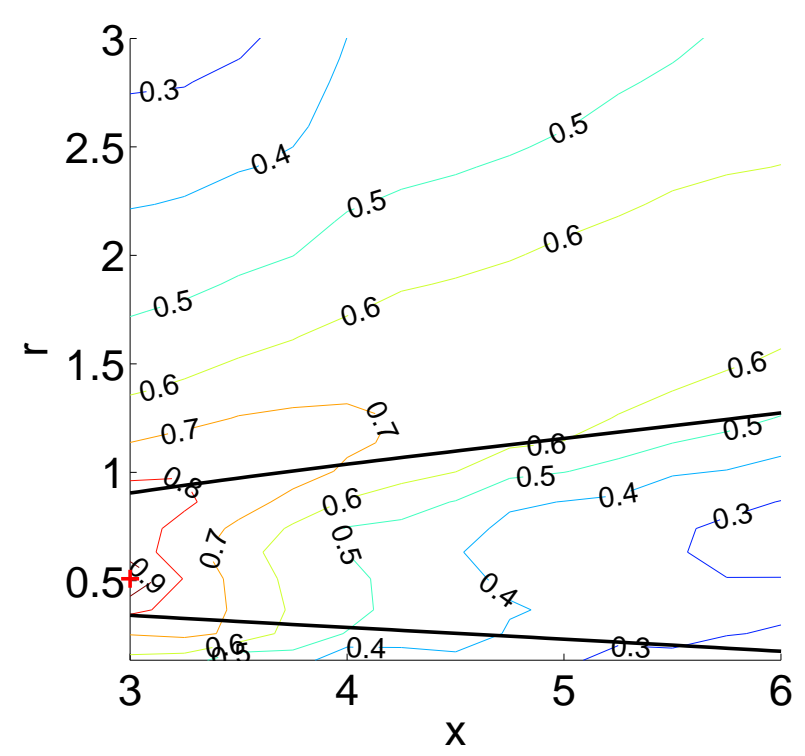

(b)

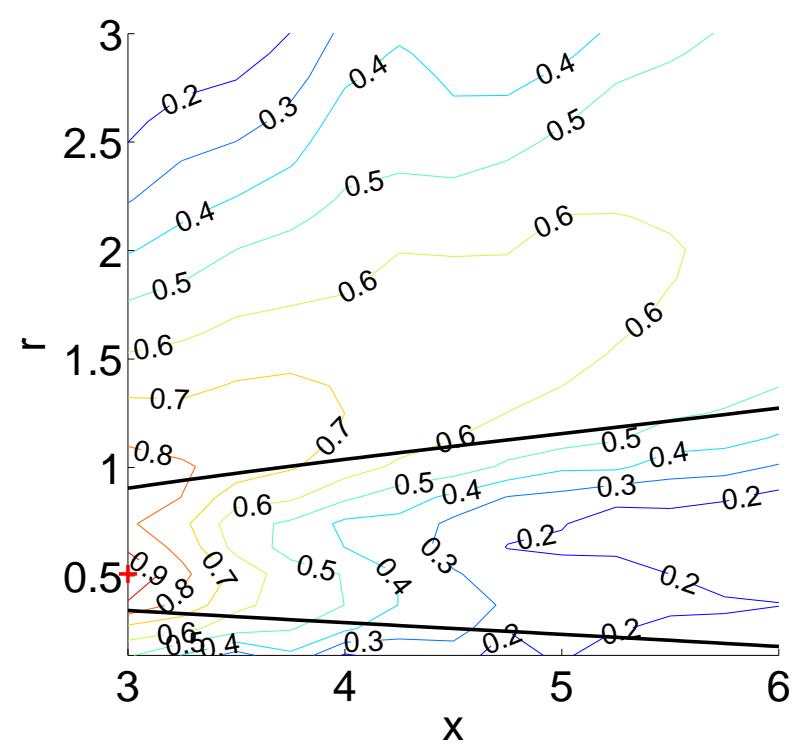

(c)

Figure 11. Contour of peak cross-correlation and peak coherence for several output positions. Input at $(x, r)=(3.0,0.51)$ (indicated by cross). Black line indicates limits of mixing layer. (a) Peak cross-correlation using PSE transfer functions. (b) Peak cross-correlation using empirical transfer functions. (c) Peak coherence. 


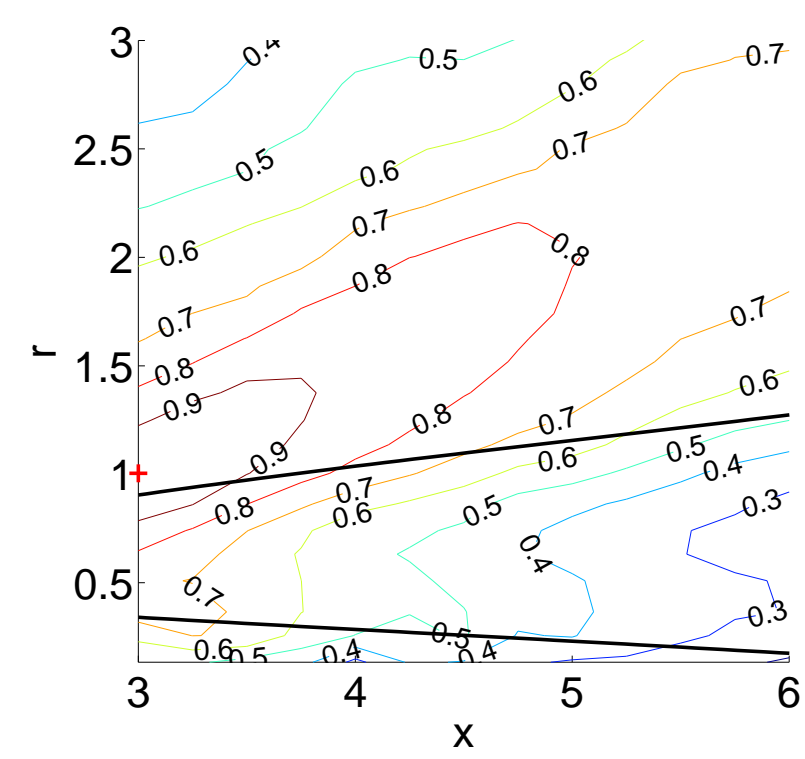

(a)

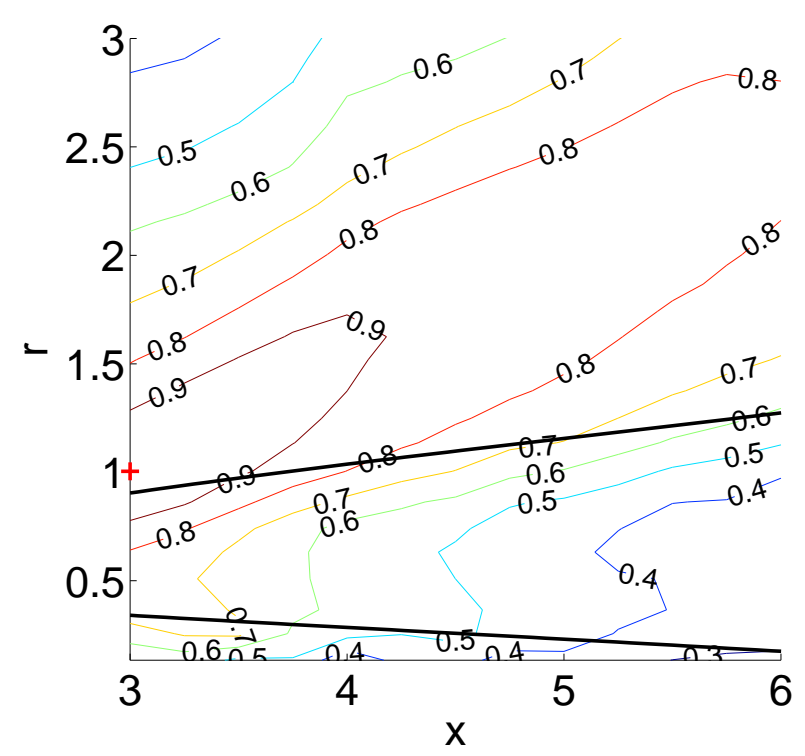

(b)

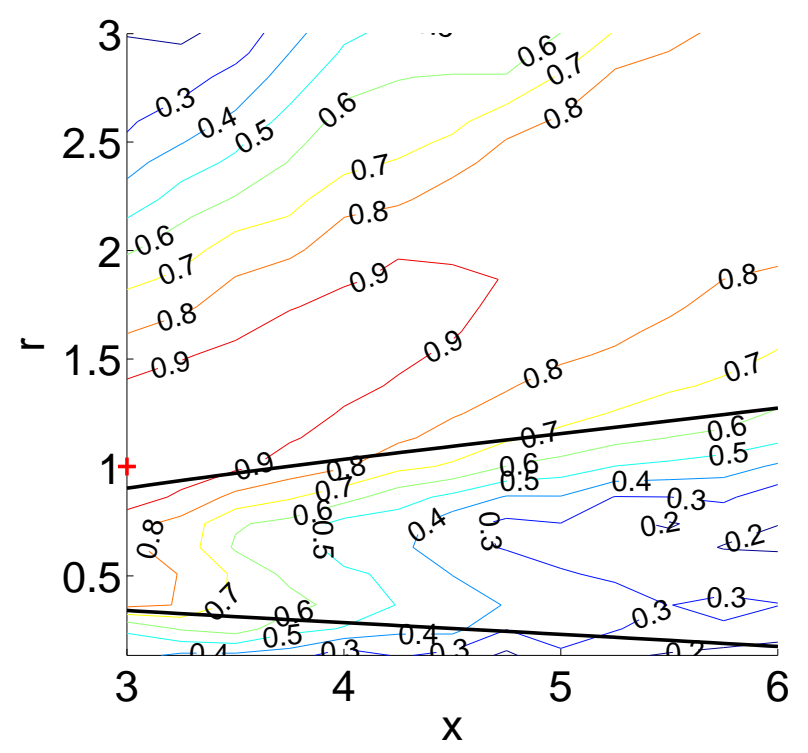

(c)

Figure 12. Contour of peak cross-correlation and peak coherence for several output positions. Input at $(x, r)=(3.0,1.0)$ (indicated by cross). Black line indicates limits of mixing layer. (a) Peak cross-correlation using PSE transfer functions. (b) Peak cross-correlation using empirical transfer functions. (c) Peak coherence. 


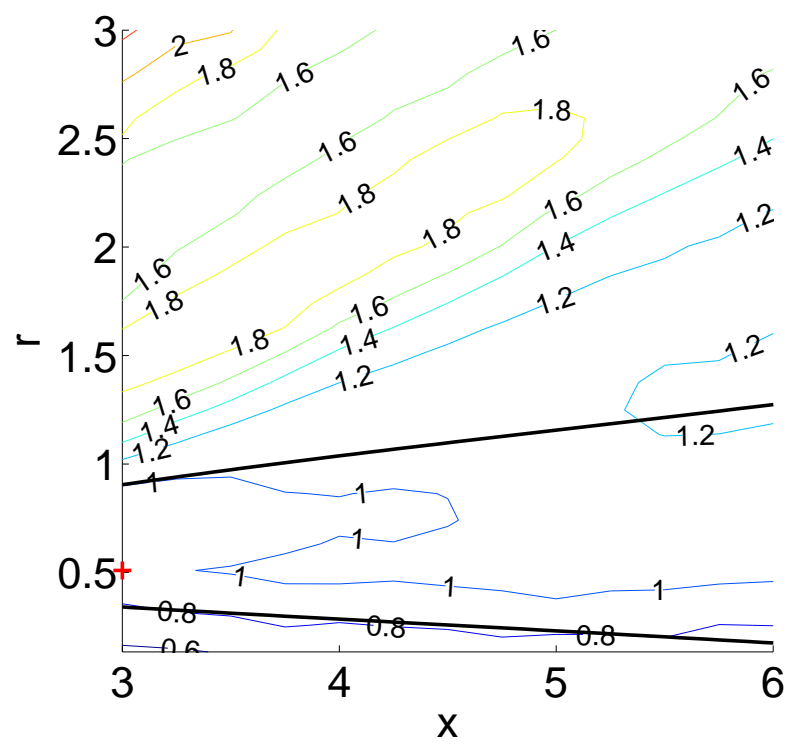

(a)

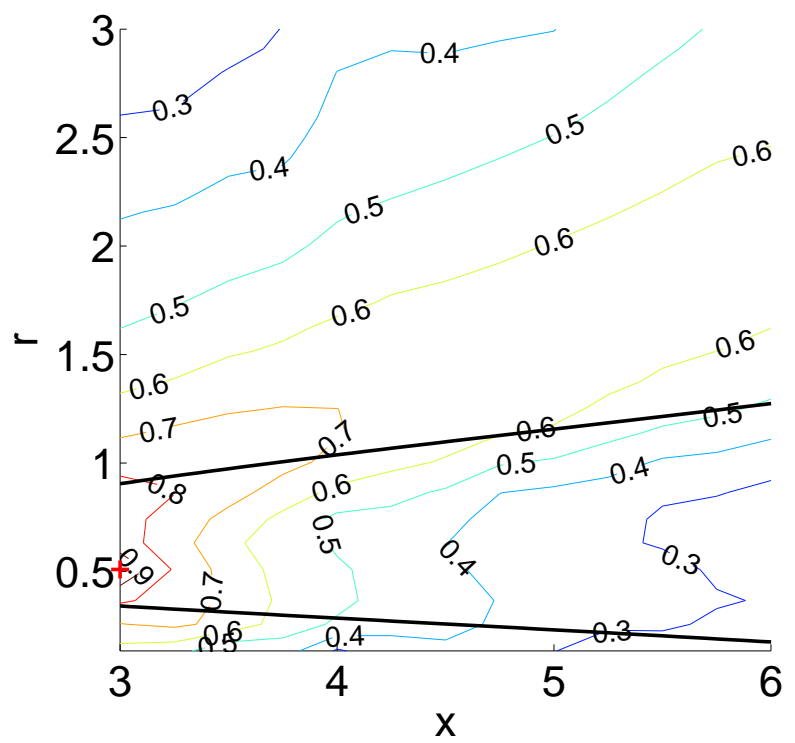

(b)

Figure 13. Ratio of root mean square (RMS) of pressure predicted by transfer function to RMS of pressure of LES. Input at $(x, r)=(3.0,0.51)$ (indicated by cross). Black line indicates limits of mixing layer. (a) RMS using PSE transfer functions. (b) RMS using empirical transfer functions.

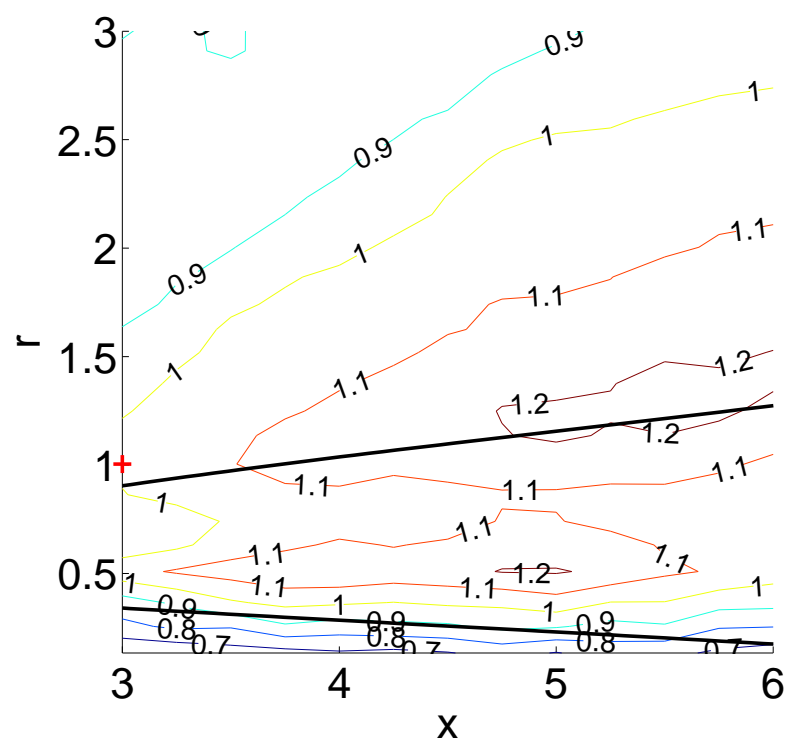

(a)

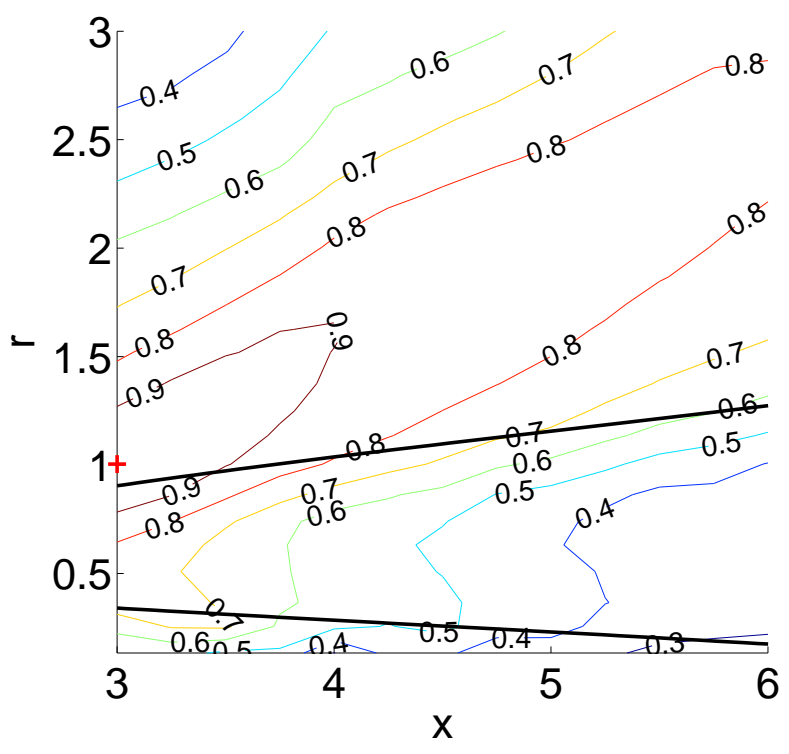

(b)

Figure 14. Ratio of root mean square (RMS) of pressure predicted by transfer function to RMS of pressure of LES. Input at $(x, r)=(3.0,1.0)$ (indicated by cross). Black line indicates limits of mixing layer. (a) RMS using PSE transfer functions. (b) RMS using empirical transfer functions. 